\title{
Efecto del Tai Chi sobre la masa y fuerza muscular en personas en proce- so de envejecimiento. Una revisión sistemática
}

\section{Effect of Tai Chion muscle mass and strength in aging people. A systematic review}

\author{
Jimena Valeria Aguilar-Curiel, ${ }^{1}$ Doris Marisol Rodríguez-Castro ${ }^{1}$ y Víctor Manuel \\ Mendoza-Núñez ${ }^{2}$
}

\author{
1 Alumna del Diplomado en Investigación Clínica y Epidemiológica, FES Zaragoza, UNAM \\ ${ }^{2}$ Coordinador Académico del Diplomado de Investigación Clínica y Epidemiológica, FES Zaragoza, UNAM
}

\section{RESUMEN}

Introducción. El envejecimiento es un proceso gradual y adaptativo que inicia en la quinta década de la vida, propicia cambios anatómicos y fisiológicos que incrementan el riesgo de enfermedades crónicas no transmisibles, entre las cuales destaca la sarcopenia. En este sentido, el ejercicio físico es una de las mejores opciones para prevenir y controlar dicha enfermedad. Al respecto el Tai Chi (TC) es uno de los ejercicios recomendados para dicho fin, sin embargo, los resultados publicados son limitados. Objetivo. Presentar una síntesis de la evidencia científica relativa al efecto del TC sobre la masa y fuerza muscular en personas en proceso de envejecimiento, a través de una revisión sistemática. Método. Se llevó a cabo una búsqueda de estudios en PubMed, Embase, Scopus, Web of Science, LILACS, SciELO, TESIUNAM y Epistemonikos acorde con los lineamientos de PRISMA. Se analizaron los estudios seleccionados hasta el 8 de enero de 2021. Resultados. Un total de 489 artículos fueron identificados y 25 estudios cumplieron con los criterios de elegibilidad, para la revisión sistemática. Respecto a la valoración de la fuerza muscular, no se reportan diferencias estadísticamente significativas entre la práctica del TC con otros tipos de ejercicio. Con relación a la masa muscular solo en dos estudios se midió esta variable, de los cuales en uno se encontró un efecto positivo sobre la masa muscular y en otro se observó una disminución estadísticamente significativa después del entrenamiento del TC. Conclusión. Los resultados sugieren que la práctica del TC durante 8 semanas o más tiene un efecto significativo sobre la fuerza de miembros superiores, similar al observado con la práctica del ejercicio físico de resistencia. No hay evidencias científicas suficientes con respecto al efecto del entrenamiento del TC sobre la masa muscular.

Palabras clave: Tai Chi, sarcopenia, fuerza muscular, edad avanzada.
Abstract

Introduction. Aging is a gradual and adaptive process that begins in the fifth decade of life. It triggers anatomical and physiological changes that increase the risk of chronic non-communicable diseases, among which sarcopenia stands out. In this sense, physical exercise is one of the best options to prevent and control this disease. In this regard, TaiChi (TC) is one of the recommended exercises for this purpose. However, the published results are limited. Objective. To present a synthesis of the scientific evidence regarding the effect of TC on muscle mass and strength in aging people through a systematic review. Method. A search for studies in PubMed, Embase, Scopus, Web of Science, LILACS, SciELO, TESIUNAM and Epistemonikos was carried out according to PRISMA guidelines. Selected studies up to January 8, 2021 were analyzed. Results. A total of 489 articles were identified and 25 studies met the eligibility criteria for the systematic review. Concerning the assessment of muscle strength, no statistically significant differences are reported between the practice of TC and other types of exercise. Regarding muscle mass, this variable was only measured in two studies, of which a positive effect on muscle mass was found in one and a statistically significant decrease was observed after TC training. Conclusion. The results suggest that the practice of TC for 8 weeks or more has a significant effect on the strength of the upper limbs, similar to that observed with the practice of resistance physical exercise. There is insufficient scientific evidence regarding the effect of TC training on muscle mass.

Key words: Tai Chi, sarcopenia, muscle strength, aged.
Correspondencia: Víctor Manuel Mendoza-Núñez E.mail: mendovic@unam.mx

Artículo recibido: 4 de octubre de 2021

Artículo aceptado: 3 de noviembre de 2021
Aguilar-Curiel JV, Rodríguez-Castro DM y Mendoza-Núñez VM Efecto del Tai Chi sobre la masa y fuerza muscular en personas en proceso de envejecimiento. Una revisión sistemática. CyRS. 2021; 3(2):47-67.

DOI: https://doi.org/10.22201/fesz.26831422e.2021.3.2.6 


\section{INTRODUCCIÓN}

El envejecimiento es un proceso gradual y adaptativo que inicia en la quinta década de la vida, caracterizado por la disminución de la reserva y respuesta biológica ante las exigencias para mantener o recuperar la homeostasis, debido a las modificaciones morfológicas, psicológicas, funcionales y bioquímicas que origina el paso del tiempo sobre los seres vivos, caracterizada por una disminución progresiva de la capacidad de adaptación ante los cambios, afectando la eficiencia de las funciones fisiológicas e incremento en la incidencia de la morbilidad, tanto de enfermedades infecciosas y crónicas no degenerativas (ECNT). ${ }^{1,2}$ En este sentido, el envejecimiento se asocia con un incremento en la incidencia de fragilidad y deterioro osteomuscular, emocional y mental conforme aumenta la edad durante el envejecimiento. Una de las ECNT de mayor prevalencia y repercusiones durante el envejecimiento y la vejez es la sarcopenia, caracterizada por la disminución significativa de la masa y fuerza muscular, así como de la funcionalidad física e incremento en la frecuencia de fragilidad. ${ }^{3} \mathrm{Al}$ respecto, desde 2016 la sarcopenia es considerada como una enfermedad, ${ }^{4}$ la prevalencia es del $10 \%$ en adultos mayores de 60 años, aumentando hasta un $50 \%$ en personas mayores de 80 años. ${ }^{5,6}$ En este contexto, en el marco de la transición demográfica, el envejecimiento poblacional representa un gran reto para la salud pública mundial, incluyendo México. ${ }^{7}$

Respecto a la fisiopatología de la sarcopenia, es importante señalar que a partir de los 30 años hay una pérdida muscular de $0.1-0.5 \%$ por año (de un 3 - $8 \%$ por década), por lo que la prevalencia de esta enfermedad puede ser hasta de un $30 \%$ después de los 60 años y del $50 \%$ después de los 80 años, ${ }^{8,9}$ esta disminución de masa muscular está acompañada de una importante reducción de fuerza. ${ }^{10}$ La pérdida de tejido y disfunción contráctil de la sarcopenia se asocia a desórdenes endócrinos y metabólicos, teniendo como resultado final una inflamación sistémica relacionada con la edad, denominada inflammaging, además del estrés oxidante (EOx) que se presenta con el envejecimiento. ${ }^{8}$ Estas alteraciones son progresivas e inevitables, pero se ha demostrado que el ritmo de degeneración se puede modificar con la realización del ejercicio físico moderado, ${ }^{10}$ ya que la actividad física regular y programada puede ayudar a mantener o mejorar tanto las capacidades funciona- les, tales como la flexibilidad, el equilibrio, la fuerza y la agilidad o movilidad corporal, así como como el estado mental, aunado a la disminución de EOx e inflammaging. ${ }^{8-11}$

Existen diferentes tipos de ejercicio físico, uno de los más recomendados en la vejez es el Tai Chi (TC), arte marcial chino que combina la respiración diafragmática profunda con diversas posturas compuestas por movimientos suaves y gentiles. ${ }^{12}$ Estas posturas requieren el cambio del peso corporal de una pierna a la otra, provocando una mejora en la fuerza muscular del tren inferior y de los músculos flexores y en el balance. ${ }^{13,14} \mathrm{Al}$ respecto, se ha demostrado que la práctica del TC disminuye significativamente el riesgo de caídas y fracturas, aunado a una percepción bienestar y mejor calidad de vida y autoeficacia. ${ }^{15,16}$ También, se ha reportado una buena adherencia a la práctica del TC en la vejez, por ser un ejercicio físico moderado muy seguro. ${ }^{17}$

Algunos estudios han mostrado resultados positivos en cuanto a la función muscular al realizar TC, ya sea sólo o combinado con alguna actividad. Al poder mantener la función y capacidad muscular de adultos mayores, la independencia y mejora en el estilo de vida son piezas clave para lograr un envejecimiento exitoso. ${ }^{17,18}$ Estos datos son de relevancia clínica y social debido a la vulnerabilidad que esta población tiene ante la fragilidad y sarcopenia. ${ }^{19}$

En este contexto es necesario tener un conocimiento preciso respecto a los diferentes estudios realizados sobre dicha temática, por lo que una de las mejores estrategias metodológicas para dicho objetivo es la realización de revisiones sistemáticas. Por tal motivo, el propósito del presente estudio es presentar una síntesis de la evidencia científica sobre el efecto de práctica del TC sobre la masa muscular y fuerza en personas en proceso de envejecimiento a través de una revisión sistemática.

\section{Material y métodos}

Se llevó a cabo una revisión sistemática acorde con los lineamientos internacionales de PRISMA (del inglés, Preferred Reporting Items for Systematic Reviews and Meta-Analyses). ${ }^{20}$ La pregunta de inves- 
tigación se estableció siguiendo el acrónimo PICO (Población, Intervención, Comparación y Resultados) fue: (i) P, adultos de 45 años y más; (ii) I, Tai Chi; (iii) $\mathrm{C}$, ejercicio físico de otro tipo o sedentarismo; (iv) O, cambios en la masa o fuerza muscular. Aunque también se consideró incluir los estudios pre-experimentales, de cohorte y transversales analíticos. Se utilizó la siguiente combinación de términos MeSH: ("Aged" OR "Frail Elderly" OR "Middle Aged") AND ("Tai Ji" OR "Tai Chi") AND ("Muscle Strength" OR "Muscle Mass" OR "Sarcopenia"). Se realizó una búsqueda en las bases científicas de datos PubMed, Embase, Scopus, Web of Science, LILACS, SciELO, TESIUNAM y Epistemonikos. Se seleccionaron los estudios publicados hasta el 8 de enero de 2021.

\section{CRiterios y Selección de Estudios}

Se incluyeron los estudios que cumplían con los siguientes criterios: 1) ensayos clínicos aleatorizados, estudios Cuasi-experimentales, pre-experimentales y de cohorte; 2) adultos de 45 a 80 años sanos y/o con alguna condición patológica controlada; 3 ) entrenamiento de Tai Chi; 4) comparación con sedentarismo o alguna otra actividad física o medición antes de la intervención; 5) que midiera la masa muscular o la fuerza; 6 ) idioma, inglés y español.

Se excluyeron los estudios de revisión, protocolos y los que no reportaron cuantitativamente el tamaño del efecto. También se excluyeron estudios que no fueron publicados como reportes completos (extractos de conferencias, cartas al editor, reportes de caso) y publicados en idiomas diferentes a los señalados.

La selección, extracción de datos y evaluación de los estudios incluidos fue llevada a cabo por dos investigadores de forma independiente (JVAC y DMRC). Cualquier desacuerdo o inconformidad fue discutida con un tercero (VMMN) para llegar a un acuerdo. Se realizó un compendio de los estudios incluidos, en una base de datos Excel.

\section{Proceso de Selección de Estudios}

Todos los estudios fueron analizados considerando los siguientes datos: 1) autores, 2) año de publicación, 3) población de estudio, 4) tamaño de la muestra, 4) tipo, duración y frecuencia de la actividad física y 5 ) resultados.
Evaluación de Resultados

Esta revisión sistemática se enfoca en dos resultados principales; fuerza y masa muscular. Para evaluar de forma cuantitativa la masa muscular se consideró los datos reportados de la composición corporal por medio de una densitometría de energía dual (DEXA) o por medio de impedancia bioeléctrica (BIA). La evaluación para fuerza es por medio de los valores de la dinamometría.

\section{RIESGO DE SESGOS}

Se utilizó la herramienta Risk of Bias 2 (RoB 2) realizada por la Colaboración Cochrane, en la que se analizan los estudios respecto a la parcialidad de cada ensayo clínico en "bajo", "poco claro" y "alto". Se evalúan los dominios relativos generación de secuencia aleatoria (sesgo de selección); ocultamiento de la asignación (sesgo de selección); cegamiento de participantes y personal (sesgo de pertinencia); cegamiento de la evaluación de resultados (sesgo de detección); datos de resultado incompletos (sesgo de deserción); informes selectivos (sesgo de informes) y otros sesgos.

\section{Resultados}

Siguiendo la estrategia de búsqueda se encontraron 489 estudios (PubMed: 69; Scopus: 58; Embase: 338; Epistemonikos: 17; LILACS: 6; TESIUNAM: 1). Después de excluir duplicados se revisaron 415 y fueron excluidos 370 después de revisar títulos y resúmenes (Figura 1).

\section{SELECCIÓN DE ESTUDIOS}

De los 45 estudios que cumplieron los criterios de inclusión y fueron revisados de texto completo, se excluyeron 20 que no cumplieron los criterios de elegibilidad (Apéndice A).

\section{Características de lOS estudios}

De los 25 estudios incluidos en el análisis sumaron un total de 2440 personas en proceso de envejecimiento. En cinco artículos la práctica de TC tuvo una duración 16 semanas, ${ }^{21-25}$ y la frecuencia más común fue de tres veces por semana $(n=11) .{ }^{15,21,26-34}$ En 16 estudios se valoró la fuerza muscular del tren infe- 
rior, ${ }^{21,24-31,33,35-40,}$ en 6 la fuerza muscular del tren superior ${ }^{22,23,32,41-43,2}$ en el tren superior e inferior. ${ }^{15,34}$ Del total de estudios seleccionados, en 3 se llevó a cabo una evaluación de la masa musculoesquelética por medio de impedancia bioeléctrica (BIA). ${ }^{23,42,44}$ En 15 artículos, la comparación se llevó a cabo con un grupo control, en los cuales se les solicitó a los participantes que no cambiaran su rutina o eran personas sedentarias (Cuadro 1).
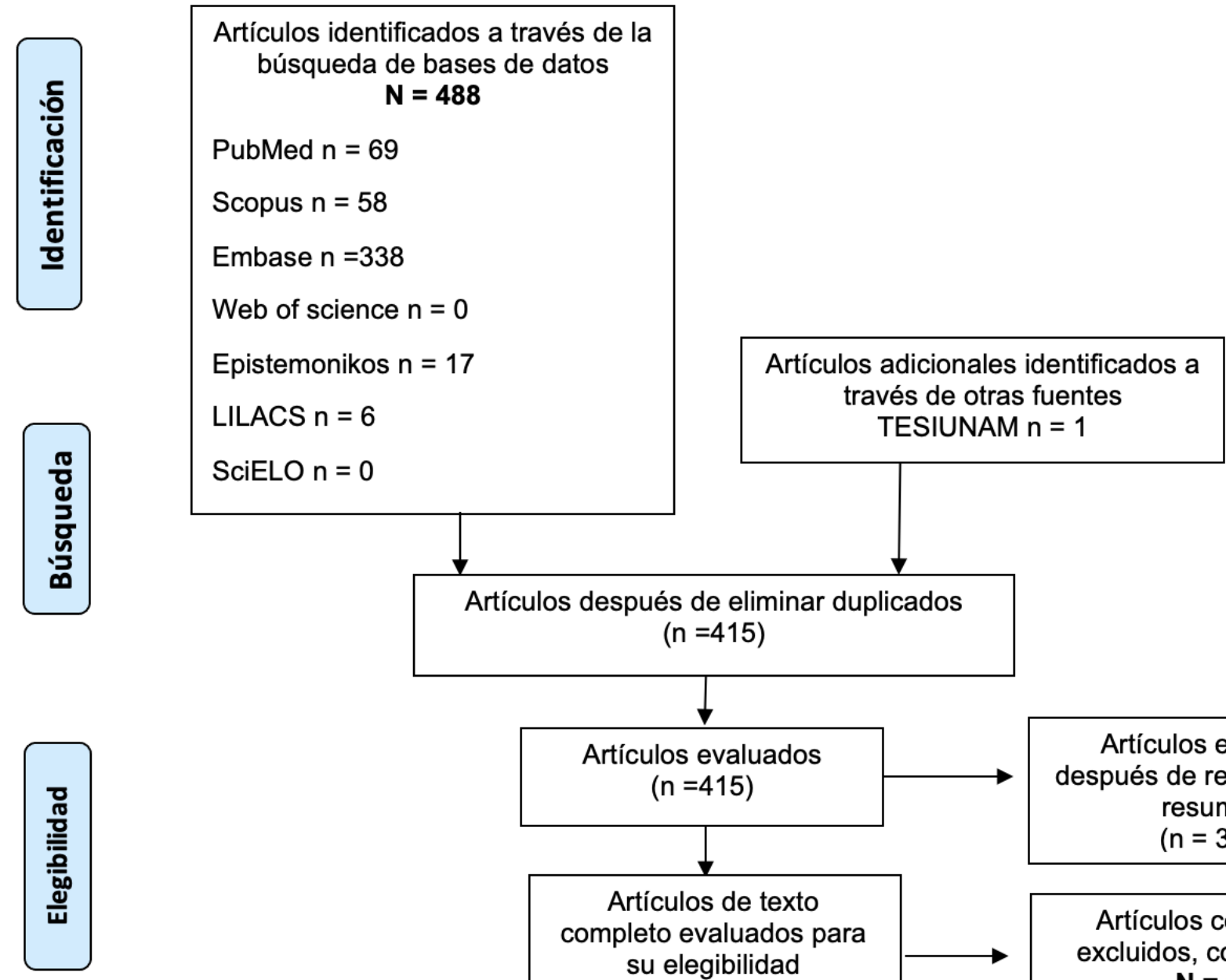

Artículos excluidos después de revisar título y resumen $(n=370)$

Artículos de texto completo evaluados para su elegibilidad $(n=45)$

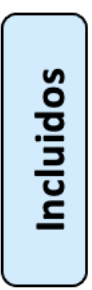

Estudios incluidos en la síntesis cualitativa (Revisión Sistemática) $(n=25)$
Artículos completos excluidos, con razones

$$
\mathbf{N}=\mathbf{2 0}
$$

- No miden fuerza de manera cuantitativa $(n=11)$

- El estudio no está completo o es un protocolo $(n=7)$

- Publicado en idioma coreano $(n=1)$

- Es una revisión sistemática $(n=1)$

Figura 1. Diagrama de flujo de los estudios seleccionados para la revisión sistemática. 


\section{Apéndice A. Estudios revisados de texto completo excluidos}

\section{Referencia}

Razón de exclusión

1. Son et al. (2016). Comparison of 2 different exercise approaches: tai chi versus otago, in community-dwelling older women. ${ }^{48}$

2. Wu (2008). Muscle action pattern and knee extensor strength of older tai chi exercisers. ${ }^{49}$

3. Chen et al. (2012). Effects of 12-week tai chi training on soleus $\mathrm{H}$-reflex and muscle strength in older adults: a pilot study. ${ }^{50}$

4. Murphy et al. (2008). Effects of 5-form, Yang Style tai chi on older females who have or are at risk for developing osteoporosis. ${ }^{51}$

5. Qi et al. (2020). Tai chi combined with resistance training for adults aged 50 years and older: a systematic review. ${ }^{2}$

6. Gallagher et al. (2003). Tai Chi Chuan and Qigong: physical and mental practice for functional mobility. ${ }^{53}$

7. Kuo et al. (2014). The comparison of impact of circuit exercise training and tai-chi exercise on multiple fracture-related risk factors in postmenopausal osteopenic women. ${ }^{54}$

8. Solloway et al. (2016). An evidence map of the effect of tai chi on health outcomes..$^{55}$

9. Callahan et al. (2016). Evaluation of tai chi program effectiveness for people with arthritis in the community: a randomized controlled trial. ${ }^{56}$

10. Gow et al. (2017). Can Tai Chi training impact fractal stride time dynamics, an index of gait health, in older adults? Cross-sectional and randomized trial studies. ${ }^{57}$

11. Zhou et al. (2016). Effects of 24-week tai chi exercise on the knee and ankle proprioception of older women. ${ }^{45}$
No mide fuerza muscular y está enfocado a riesgo de caída.

No mide fuerza muscular.

No evalúa fuerza ni masa muscular.

No evalúa la fuerza muscular.

Es una revisión sistemática, los resultados no son específicos.

No es un estudio clínico.

No es un estudio clínico.

No es un estudio clínico.

No mide fuerza muscular, se enfoca en medición de dolor o rigidez.

El artículo no mide fuerza muscular. 


\section{Apéndice A. Estudios revisados de texto completo excluidos}

12. Yildirim (2016). Tai Chi vs. combined exercise prescription: a comparison of their effects on factors related to falls. ${ }^{58}$

13. Lee et al. (2006). Comparison of effects among Tai-Chi exercise, aquatic exercise, and a self-help program for patients with knee osteoarthritis. ${ }^{59}$

14. Li et al. (2018). Effectiveness of a therapeutic Tai Ji Quan intervention vs. a multimodal exercise intervention to prevent falls among older adults at high risk of falling: a randomized clinical trial. ${ }^{60}$

15. Li et al. (2019). Effectiveness of Tai Ji Quan vs multimodal and stretching exercise interventions for reducing injurious falls in older adults at high risk of falling: follow-up analysis of a randomized clinical trial. ${ }^{61}$

16. Rosado-Pérez et al. (2013). Effect of tai chi versus walking on oxidative stress in Mexican older adults. ${ }^{62}$

17. Sun et al. (2019). Effects of Tai Chi Chuan and brisk walking exercise on balance ability in elderly women: a randomized controlled trial. ${ }^{63}$

18. Kienle et al. (2020). A multi-centre, parallelgroup, randomised controlled trial to assess the efficacy and safety of eurhythmy therapy and tai chi in comparison with standard care in chronically ill elderly patients with increased risk of falling a trial protocol. ${ }^{64}$

19. Milhay et al. (2006). The effect of tai chi inspired exercise compared to strength training: a pilot study of elderly retired community dwellers. ${ }^{65}$

20. Pereira et al. (2008). Effects of Tai Chi Chuanon knee extensor muscle strength and balance in elderly women. ${ }^{66}$
No mide fuerza muscular.

Artículo en idioma coreano.

No mide fuerza muscular, está enfocado en miedo a caídas.

El estudio está enfocado a medir riesgo en caídas.

No mide masa ni fuerza muscular.

No mide masa ni fuerza muscular.

El estudio no se enfoca en medir la fuerza muscular.

No mide masa ni fuerza muscular.

No mide la fuerza muscular con dinamómetro. 


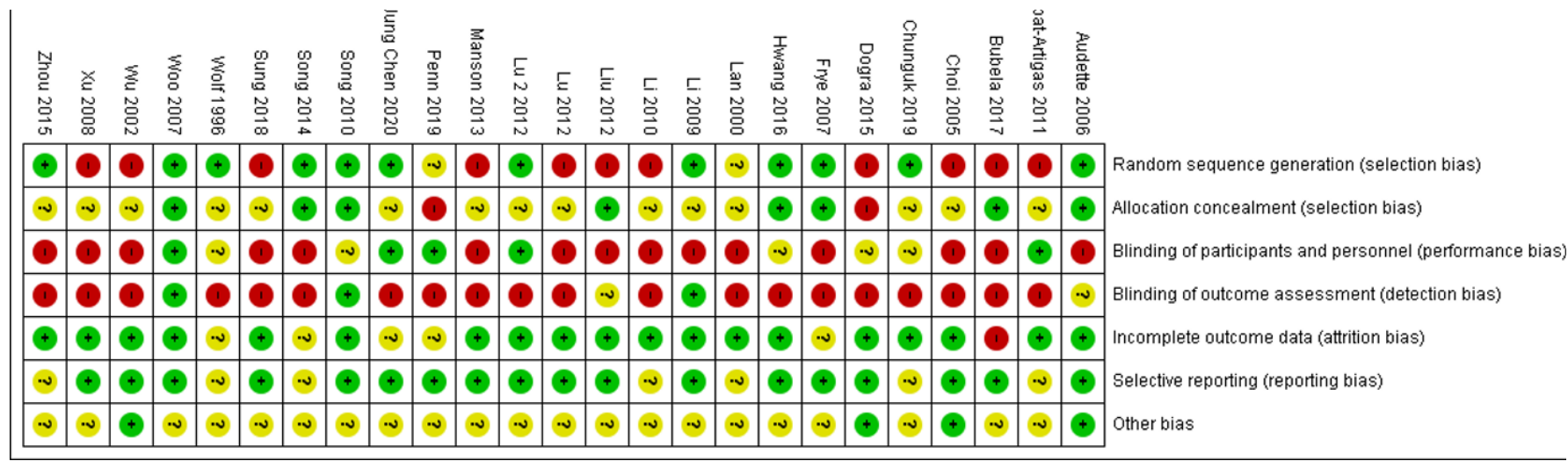

Figura 2. Tabla resumida de riesgo de sesgos.

Random sequence generation (selection bias)

Allocation concealment (selection bias)

Blinding of participants and personnel (performance bias)

Blinding of outcome assessment (detection bias)

Incomplete outcome data (attrition bias)
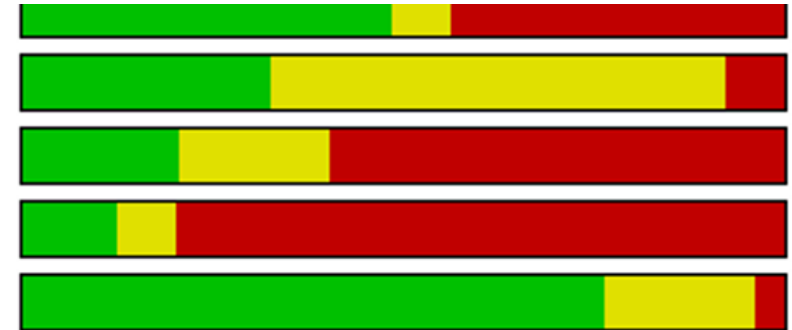

\section{Selective reporting (reporting bias) \\ Other bias}
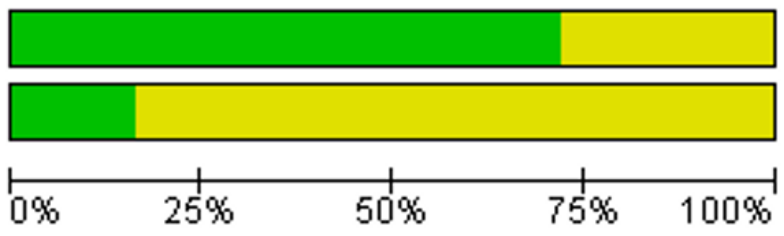

Low risk of bias

Figura 3. Gráfica de riesgo de sesgos

\section{EVALUACIÓN DE RIESGO DE SESGOS}

En las figuras 2 y 3 se muestra el sesgo de los estudios. Al respecto en doce estudios (48\%) se observó que tenían 3 dominios con "bajo riesgo" y nueve (36\%) tuvieron tres dominios con "alto riesgo".

\section{FUERZA MUSCULAR}

En todos los artículos se evaluó por medio de dinamometría en diversos músculos en el tren inferior; cadera (flexor, extensor, abductor, aductor), rodilla (extensor, flexor), músculo iliopsoas, cuádriceps femoral, isquiotibiales, tibial anterior y tobillo (dorsiflexión y flexión plantar) y en el tren superior con fuerza de agarre y flexión de bíceps (curl de bíceps). El segundo resultado a evaluar fue la masa muscular se utilizó la BIA y fue por medio de la masa musculoesquelética. 
Cuadro 1. Características y evidencias de los estudios sobre el efecto del Tai Chi sobre fuerza y masa muscular incluidos en la revisión sistemática

\begin{tabular}{|c|c|c|c|c|c|c|c|}
\hline $\begin{array}{l}\text { Autor/ } \\
\text { año }\end{array}$ & Objetivo & Población & $\begin{array}{l}\text { Tipo de } \\
\text { Tai Chi }\end{array}$ & $\begin{array}{c}\text { Duración, } \\
\text { frecuencia y } \\
\text { tiempo de Tai Chi }\end{array}$ & $\begin{array}{c}\text { Diseño de } \\
\text { investigación }\end{array}$ & Medición & Resultados \\
\hline $\begin{array}{l}\text { Chen } \\
\text { et al. } \\
(2020)^{27}\end{array}$ & $\begin{array}{l}\text { Determinar el } \\
\text { efecto sobre la } \\
\text { fuerza muscular del } \\
\text { Tai Chi simplificado } \\
\text { (sTC) en compar- } \\
\text { ación con el Tai Chi } \\
\text { tradicional (tTC). }\end{array}$ & $\begin{array}{l}n=28 \\
65 \text { a } 80 \text { años. } \\
H: 14 \\
\text { M: } 14\end{array}$ & $\begin{array}{l}\text { Tai Chi } \\
\text { simplificado } \\
\text { (sTC) } \\
\text { Forma } 8 \\
\text { movimientos } \\
\text { sencillos. } \\
\text { Tradicional } \\
\text { (tTC) forma } \\
24, \text { estilo } \\
\text { Yang. }\end{array}$ & $\begin{array}{l}30 \text { minutos, } 3 \\
\text { veces por se- } \\
\text { mana durante } 8 \\
\text { semanas. }\end{array}$ & $\begin{array}{l}\text { Ensayo clínico } \\
\text { aleatorizado } \\
\text { Grupos: Tai Chi } \\
\text { Tradicional (†TC) } \\
\text { forma } 24 \text { estilo } \\
\text { yang ( } n=14 \text { ) } \\
\text { sTC: Forma } 24, \\
\text { estilo Yang simplifi- } \\
\text { cada ( } n=14 \text { ) }\end{array}$ & $\begin{array}{l}\text { Dinamómetro isocinético: } \\
\text { - Flexor de cadera derecho e } \\
\text { izquierdo (FCD y FCI) } \\
\text { - Extensor de cadera derecho e } \\
\text { izquierdo (ECD y ECI) } \\
\text { - Abductor de cadera derecho e } \\
\text { izquierdo (AbCD y AbCI) } \\
\text { - Aductor de cadera derecho e } \\
\text { izquierdo (AdCD y AdCI) } \\
\text { - Extensor de rodilla (ER) } \\
\text { - Flexor de rodilla derecho e izqui- } \\
\text { erdo (FRD y FRD) } \\
\text { - Dorsiflexión de tobillo derecho e } \\
\text { izquierdo (DFTD y DFTI) } \\
\text { - Flexión plantar de tobillo dere- } \\
\text { cho e izquierdo (FPTD y FPTI) }\end{array}$ & $\begin{array}{l}\text { †TC y sTC: mostraron } \\
\text { un aumento sig- } \\
\text { nificativo después } \\
\text { del entrenamiento: } \\
\text { (FCD, } \mathrm{p}=0.032) ;(\mathrm{FCl} \text {, } \\
\mathrm{p}=0.033) ;(\mathrm{AbCl}, \\
\mathrm{p}=0.001) ;(\mathrm{DFTD}, \\
\mathrm{p}=0.001) ;(\text { (DFTI, } \\
\mathrm{p}=0.002) \text {. } \\
\text { Sin diferencias signifi- } \\
\text { cativas entre grupos. }\end{array}$ \\
\hline $\begin{array}{l}\text { Penn } \\
\text { et al. } \\
(2019)^{28}\end{array}$ & $\begin{array}{l}\text { Determinar el } \\
\text { efecto del Tai Chi } \\
\text { individualizado (iTC) } \\
\text { en compasión con } \\
\text { Tai Chi tradicional } \\
\text { (tTC) sobre el bal- } \\
\text { ance funcional en } \\
\text { la fuerza de extrem- } \\
\text { idades inferiores. }\end{array}$ & $\begin{array}{l}n=50 \\
65 \text { a } 80 \text { años. } \\
H: 7 \\
M: 43\end{array}$ & $\begin{array}{l}\text { Tai Chi Indi- } \\
\text { vidualizado } \\
\text { (iTC) (forma } \\
24 \text { dividida } \\
\text { por dificultad, } \\
\text { estilo Yang) }\end{array}$ & $\begin{array}{l}30 \text { minutos, } 3 \\
\text { veces por se- } \\
\text { mana durante } 8 \\
\text { semanas. }\end{array}$ & $\begin{array}{l}\text { Cuasi-experimen- } \\
\text { tal } \\
\text { Grupos: } \\
\text { TC Tradicional } \\
\text { (tTC) forma 24, } \\
\text { estilo Yang } \\
\text { (n=15) } \\
\text { Grupo control } \\
\text { (GC) } \\
\text { (n=15) } \\
\text { iTC (n=20) }\end{array}$ & $\begin{array}{l}\text { Dinamómetro isocinético: } \\
\text { - Flexor de cadera derecho e } \\
\text { izquierda (FCD, FCl) } \\
\text { - Extensor de cadera derecho e } \\
\text { izquierda (ECD, ECI) } \\
\text { - Abductor de cadera derecho e } \\
\text { izquierdo (AbCD, AbCI) } \\
\text { - Aductor de cadera derecho e } \\
\text { izquierdo (AdCD, AdCI) } \\
\text { - Extensor de rodilla derecho e } \\
\text { izquierdo (ERD, ERI) } \\
\text { - Flexor de rodilla derecho e izqui- } \\
\text { erdo (FRD, FRI) } \\
\text { - Dorsiflexión de tobillo derecho e } \\
\text { izquierdo (DFTD, DFTI) } \\
\text { - Flexión plantar de tobillo dere- } \\
\text { cho e izquierdo (FPTD, FPTI) }\end{array}$ & $\begin{array}{l}\text { iTC y tTC mostraron un } \\
\text { aumento estadística- } \\
\text { mente significativo de } \\
\text { fuerza muscular } \\
\text { (p <0.05) } \\
\text { iTC mostró un aumen- } \\
\text { to estadísticamente } \\
\text { significativo en com- } \\
\text { paración con tTC en } \\
\text { AbCD, AdCD, AdCl, } \\
\text { ERD, DFTD, FPTD, FPTI } \\
\text { (p<0.001). }\end{array}$ \\
\hline
\end{tabular}


Cuadro 1. Características y evidencias de los estudios sobre el efecto del Tai Chi sobre fuerza y masa muscular incluidos en la revisión sistemática

\begin{tabular}{|c|c|c|c|c|c|c|c|}
\hline $\begin{array}{l}\text { Autor/ } \\
\text { año }\end{array}$ & Objetivo & Población & $\begin{array}{c}\text { Tipo de } \\
\text { Tai Chi }\end{array}$ & $\begin{array}{c}\text { Duración, } \\
\text { frecuencia y } \\
\text { tiempo de Tai Chi }\end{array}$ & $\begin{array}{c}\text { Diseño de } \\
\text { investigación }\end{array}$ & Medición & Resultados \\
\hline $\begin{array}{l}\text { Sung } \\
\text { et al. } \\
(2019)^{29}\end{array}$ & $\begin{array}{l}\text { Evaluar el efecto } \\
\text { del Tai Chi simplifi- } \\
\text { cado individualiza- } \\
\text { do sobre la fuerza } \\
\text { del extensor de } \\
\text { rodilla de adultos } \\
\text { mayores de una } \\
\text { comunidad. }\end{array}$ & $\begin{array}{l}n=10 \\
62 \text { a } 87 \text { años. } \\
H: 8 \\
M: 2\end{array}$ & $\begin{array}{l}\text { Tai Chi (TC) } \\
\text { (forma } 24 \\
\text { simplificada, } \\
\text { estilo Yang) }\end{array}$ & $\begin{array}{l}30 \text { minutos, } 3 \\
\text { veces por } \\
\text { semana durante } \\
8 \text { semanas. }\end{array}$ & Pre-experimental & $\begin{array}{l}\text { Dinamómetro isociné- } \\
\text { tico: } \\
\text { - Extensor de rodilla } \\
\text { (ER) }\end{array}$ & $\begin{array}{l}\text { TC: Mostró un aumento } \\
\text { estadísticamente significa- } \\
\text { tivo en la fuerza en extremi- } \\
\text { dades inferiores ( } \mathrm{p}=0.002) \text {. }\end{array}$ \\
\hline $\begin{array}{l}\text { Bubela } \\
\text { et al. } \\
(2019)^{21}\end{array}$ & $\begin{array}{l}\text { Evaluar el efecto } \\
\text { de la práctica del } \\
\text { Tai Chi sobre el } \\
\text { riesgo de caídas } \\
\text { (fuerza, movilidad } \\
\text { funcional). }\end{array}$ & $\begin{array}{l}n=30 \\
55 \text { a } 86 \text { años. } \\
H: 15 \\
M: 15\end{array}$ & $\begin{array}{l}\text { Tai Chi (TC) } \\
\text { (no mencio- } \\
\text { nan la forma } \\
\text { ni el estilo) }\end{array}$ & $\begin{array}{l}60 \text { minutos, } 3 \text { vec- } \\
\text { es por semana } \\
\text { por } 16 \text { semanas. }\end{array}$ & $\begin{array}{l}\text { Cuasi-experimental } \\
\text { Grupos: } \\
\text { TC (n=26) } \\
\text { Grupo control } \\
\text { (GC) } \\
(n=14)\end{array}$ & $\begin{array}{l}\text { Dinamómetro isociné- } \\
\text { tico: } \\
\text { - Extensor de rodilla } \\
\text { derecho e izquierdo } \\
\text { (ERD, ERI) }\end{array}$ & $\begin{array}{l}\text { TC: mostró una diferencia } \\
\text { estadísticamente significa- } \\
\text { tiva en ERD en comparación } \\
\text { con el GC ( } p=0.042) \text {. }\end{array}$ \\
\hline $\begin{array}{l}\text { Hwang } \\
\text { et al. } \\
(2016)^{43}\end{array}$ & $\begin{array}{l}\text { Determinar el } \\
\text { efecto del Tai Chi } \\
\text { guiado en casa en } \\
\text { comparación con } \\
\text { el entrenamiento de } \\
\text { extremidades infe- } \\
\text { riores sobre la fun- } \\
\text { cionalidad física en } \\
\text { adultos mayores con } \\
\text { riesgo de caídas. }\end{array}$ & $\begin{array}{l}n=456 \\
60 \text { a } 80 \text { años. } \\
H: 152 \\
M: 304\end{array}$ & $\begin{array}{l}\text { Tai Chi (TC) } \\
\text { (forma 18, } \\
\text { estilo Yang). }\end{array}$ & $\begin{array}{l}60 \text { minutos, } 1 \\
\text { vez por semana } \\
\text { durante } 24 \text { sema- } \\
\text { nas. }\end{array}$ & $\begin{array}{l}\text { Ensayo clínico } \\
\text { aleatorizado sim- } \\
\text { ple ciego } \\
\text { Grupos: } \\
\text { Entrenamiento } \\
\text { de extremidades } \\
\text { inferior inferiores } \\
\text { (LEI) ( } n=228) \text {. } \\
\text { TC }(n=228)\end{array}$ & $\begin{array}{l}\text { Dinamómetro: } \\
\text { - Fuerza de agarre } \\
\text { (FA) }\end{array}$ & $\begin{array}{l}\text { TC y LEl: mostraron un } \\
\text { aumento estadísticamente } \\
\text { significativo de FA a } 6 \text { y } 18 \\
\text { meses } \\
\text { (p<0.05). } \\
\text { Sin diferencias entre grupos } \\
\text { de entrenamiento. }\end{array}$ \\
\hline $\begin{array}{l}\text { Zhou } \\
\text { et al. } \\
(2016)^{35}\end{array}$ & $\begin{array}{l}\text { Evaluar el efecto } \\
\text { de la práctica del } \\
\text { Tai Chi sobre los } \\
\text { músculos inferiores, } \\
\text { en diferentes gru- } \\
\text { pos de edad. }\end{array}$ & $\begin{array}{l}n=410 \\
60 \text { a } 89 \text { años } \\
\text { G1: } 60 \text { a } 69 \\
\text { años. } \\
\text { G2: } 70 \text { a } 79 \\
\text { años. } \\
\text { G3: } 80 \text { a } 89 \\
\text { años. } \\
\text { H:218 } \\
\text { M:192 }\end{array}$ & $\begin{array}{l}\text { Tai Chi (TC) } \\
\text { (forma } 24, \\
\text { estilo Yang) }\end{array}$ & $\begin{array}{l}50 \text { minutos } \\
7 \text { veces por } \\
\text { semana, pre- } \\
\text { viamente lo } \\
\text { practicaban (3-30 } \\
\text { años). }\end{array}$ & $\begin{array}{l}\text { Transversal analíti- } \\
\text { co } \\
\text { Grupos: } \\
\text { Caminata (Cam) } \\
120 \text { minutos } \\
\text { (n=205) } \\
\text { TC }(n=195)\end{array}$ & $\begin{array}{l}\text { Dinamómetro isociné- } \\
\text { tico: } \\
\text { - Iliopsoas (II) } \\
\text { - Cuádriceps femoral } \\
\text { (CF) } \\
\text { - Tibial anterior (TA) } \\
\text { - Isquiotibiales (Is }\end{array}$ & $\begin{array}{l}\text { TC: no mostró cambios es- } \\
\text { tadísticamente significativa } \\
\text { en fuerza muscular en los } \\
\text { diferentes grupos de edad. } \\
\text { Al comparar entre grupos: } \\
\text { TC G2 y G3: fuerza muscu- } \\
\text { lar mayor en II, CF, TA e Is } \\
\text { (p<0.01) al compararlo con } \\
\text { NTC. } \\
\text { TC: mayor fuerza muscular } \\
\text { en Il en todos los grupos de } \\
\text { edad (p<0.05) al compara- } \\
\text { rlo con NTC. }\end{array}$ \\
\hline
\end{tabular}


Cuadro 1. Características y evidencias de los estudios sobre el efecto del Tai Chi sobre fuerza y masa muscular incluidos en la revisión sistemática

\begin{tabular}{|c|c|c|c|c|c|c|c|}
\hline $\begin{array}{l}\text { Autor/ } \\
\text { año }\end{array}$ & Objetivo & Población & $\begin{array}{c}\text { Tipo de } \\
\text { Tai Chi }\end{array}$ & $\begin{array}{c}\text { Duración, } \\
\text { frecuencia y } \\
\text { tiempo de Tai Chi }\end{array}$ & $\begin{array}{c}\text { Diseño de } \\
\text { investigación }\end{array}$ & Medición & Resultados \\
\hline $\begin{array}{l}\text { Dogra } \\
\text { et al. } \\
(2015)^{22}\end{array}$ & $\begin{array}{l}\text { Evaluar el efecto } \\
\text { de un programa de } \\
\text { Tai Chi de } 16 \text { sema- } \\
\text { nas para mejorar la } \\
\text { funcionalidad física } \\
\text { de adultos mayores } \\
\text { con artritis. }\end{array}$ & $\begin{array}{l}n=410 \\
60 \text { a } 89 \text { años } \\
\text { G1: } 60 \text { a } 69 \\
\text { años. } \\
\text { G2: } 70 \text { a } 79 \\
\text { años. } \\
\text { G3: } 80 \text { a } 89 \\
\text { años. } \\
\text { H:218 } \\
\text { M: } 192\end{array}$ & $\begin{array}{l}\text { Tai Chi (TC) } \\
\text { (forma 24, } \\
\text { estilo Yang) }\end{array}$ & $\begin{array}{l}50 \text { minutos } \\
7 \text { veces por } \\
\text { semana, pre- } \\
\text { viamente lo } \\
\text { practicaban (3-30 } \\
\text { años). }\end{array}$ & $\begin{array}{l}\text { Transversal analíti- } \\
\text { co } \\
\text { Grupos: } \\
\text { Caminata (Cam) } \\
120 \text { minutos } \\
\text { (n=205) } \\
\text { TC (n=195) }\end{array}$ & $\begin{array}{l}\text { Dinamómetro isocinético: } \\
\text { - lliopsoas (II) } \\
\text { - Cuádriceps femoral (CF) } \\
\text { - Tibial anterior (TA) } \\
\text { - Isquiotibiales (Is) }\end{array}$ & $\begin{array}{l}\text { TC: no mostró cambios es- } \\
\text { tadísticamente significativa } \\
\text { en fuerza muscular en los } \\
\text { diferentes grupos de edad. } \\
\text { Al comparar entre grupos: } \\
\text { TC G2 y G3: fuerza muscu- } \\
\text { lar mayor en II, CF, TA e Is } \\
\text { (p<0.01) al compararlo con } \\
\text { NTC. } \\
\text { TC: mayor fuerza muscular } \\
\text { en II en todos los grupos de } \\
\text { edad (p<0.05) al compararlo } \\
\text { con NTC. }\end{array}$ \\
\hline $\begin{array}{l}\text { Song } \\
\text { et al. } \\
(2014)^{36}\end{array}$ & $\begin{array}{l}\text { Determinar el } \\
\text { efecto del Tai Chi } \\
\text { sobre la cantidad } \\
\text { de masa muscular } \\
\text { esquelética y fuerza } \\
\text { de extremidades } \\
\text { inferiores, densidad } \\
\text { mineral ósea y fun- } \\
\text { ción de balance. }\end{array}$ & $\begin{array}{l}n=94 \\
55 \text { a } 65 \text { años. } \\
\text { M: } 94\end{array}$ & $\begin{array}{l}\text { Tai Chi (TC) } \\
\text { (estilo Chen, } \\
\text { no mencio- } \\
\text { nan forma). }\end{array}$ & $\begin{array}{l}40 \text { minutos, } \\
6 \text { veces por se- } \\
\text { mana, } 52 \text { sema- } \\
\text { nas. }\end{array}$ & $\begin{array}{l}\text { Ensayo clínico } \\
\text { aleatorizado } \\
\text { Grupos: } \\
\text { Grupo baile (CG1) } \\
\text { (n=33) } \\
\text { Grupo caminata } \\
\text { rápida (CG 2) } \\
\text { (n=30) } \\
\text { TC (n=31) }\end{array}$ & $\begin{array}{l}\text { Dinamómetro isocinético: } \\
\text { - Extensor de rodilla (ER) } \\
\text { - Extensor de cadera (EC) }\end{array}$ & $\begin{array}{l}\text { En los } 3 \text { grupos hubo un } \\
\text { aumento significativo ER y EC } \\
(p<0.05)\end{array}$ \\
\hline $\begin{array}{l}\text { Manson } \\
\text { et al. } \\
(2013)^{23}\end{array}$ & $\begin{array}{l}\text { Evaluar el efecto } \\
\text { de un programa } \\
\text { de Tai Chi sobre } \\
\text { los cambios en el } \\
\text { musculo esquelé- } \\
\text { tico (cantidad y } \\
\text { calidad) en adultos } \\
\text { mayores. }\end{array}$ & $\begin{array}{l}n=153 \\
50 \text { a } 80 \text { años. } \\
\text { Divididos } \\
\text { en diversas } \\
\text { cohortes: } \\
\text { C1: } 57 \\
\text { C2: } 60 \\
\text { C3: } 36 \\
\text { H: } 30\end{array}$ & $\begin{array}{l}\text { Tai Chi (TC) } \\
\text { (no menciona } \\
\text { la forma, es- } \\
\text { tilo Yang). }\end{array}$ & $\begin{array}{l}60 \text { minutos, } 2 \\
\text { veces por se- } \\
\text { mana durante } 16 \\
\text { semanas. }\end{array}$ & Estudio de cohorte & $\begin{array}{l}\text { Dinamómetro: } \\
\text { - Fuerza de agarre (FA) } \\
\text { - Curl de brazo (CB) } \\
\text { Analizador de composición } \\
\text { corporal } \\
\text { - Masa musculoesquelética } \\
\text { inferior (MMEl) }\end{array}$ & $\begin{array}{l}\text { FA: aumento significativo } C 1 \\
(p=0.002) \text {. } \\
\text { CB: aumento significativo } C 1 \text {, } \\
\text { C2 y } C 3 \text { ( } p<0.001) \text {. } \\
\text { FA y CB: diferencias significa- } \\
\text { tivas al combinar } C 1 \text {, C2 y C3 } \\
\text { ( } p<0.001) \text {. } \\
\text { MMEl: aumento significativo a } \\
\text { partir de } 4 \text { meses } C 1, C 2 \text { y } C 3 \\
\text { ( } p<0.05) \text {. }\end{array}$ \\
\hline
\end{tabular}


Cuadro 1. Características y evidencias de los estudios sobre el efecto del Tai Chi sobre fuerza y masa muscular incluidos en la revisión sistemática

\begin{tabular}{|c|c|c|c|c|c|c|c|}
\hline $\begin{array}{l}\text { Autor/ } \\
\text { año }\end{array}$ & Objetivo & Población & $\begin{array}{l}\text { Tipo de } \\
\text { Tai Chi }\end{array}$ & $\begin{array}{c}\text { Duración, } \\
\text { frecuencia y } \\
\text { tiempo de Tai Chi }\end{array}$ & $\begin{array}{c}\text { Diseño de } \\
\text { investigación }\end{array}$ & Medición & Resultados \\
\hline $\begin{array}{l}\text { Lu et al. } \\
(2013)^{24}\end{array}$ & $\begin{array}{l}\text { Determinar el } \\
\text { efecto del Tai } \\
\text { Chi sobre la fuerza } \\
\text { muscular en rodilla } \\
\text { en personas que lo } \\
\text { han practicado por } \\
\text { más de } 3 \text { años. }\end{array}$ & $\begin{array}{l}n=31 \\
65 \text { a } 77 \text { años. } \\
M: 31\end{array}$ & $\begin{array}{l}\text { Practicantes } \\
\text { Tai Chi (TC) } \\
\text { (debido } \\
\text { a que son } \\
\text { personas ya } \\
\text { practicantes, } \\
\text { no mencio- } \\
\text { nan forma ni } \\
\text { estilo) }\end{array}$ & $\begin{array}{l}1 \text { hora } 30 \text { minutos } \\
\text { por semana, con } \\
\text { experiencia de } 3 \\
\text { años }\end{array}$ & $\begin{array}{l}\text { Ensayo clínico aleatori- } \\
\text { zado simple ciego } \\
\text { Grupos: } \\
\text { TC (n=31) } \\
\text { Grupo control (GC) (cam- } \\
\text { inata, ejercicio ligero) } \\
(n=36)\end{array}$ & $\begin{array}{l}\text { Dinamómetro: } \\
\text { - Extensor de rodilla } \\
\text { (ER) } \\
\text { - Flexor de rodilla (FR) }\end{array}$ & $\begin{array}{l}\text { TC: mayor fuerza } \\
\text { excéntrica prome- } \\
\text { dio ER }(p=0.01) \text { y FR } \\
(p=0.03) \text {. }\end{array}$ \\
\hline $\begin{array}{l}\text { Lu et al. } \\
(2013)^{30}\end{array}$ & $\begin{array}{l}\text { Evaluar el efecto } \\
\text { de la práctica del } \\
\text { Tai Chi sobre la } \\
\text { fuerza muscular. }\end{array}$ & $\begin{array}{l}n=65 \\
60 \text { a } 80 \text { años. } \\
H: 15 \\
M: 50\end{array}$ & $\begin{array}{l}\text { Tai Chi (TC) } \\
\text { (forma 12, } \\
\text { estilo Yang). }\end{array}$ & $\begin{array}{l}60 \text { minutos, } 3 \\
\text { veces por se- } \\
\text { mana durante } 16 \\
\text { semanas. }\end{array}$ & $\begin{array}{l}\text { Ensayo clínico aleatorizado } \\
\text { Grupos: } \\
\text { TC (n= 29) } \\
\text { Grupo programa educa- } \\
\text { tivo (GC) } \\
\text { (n=36) }\end{array}$ & $\begin{array}{l}\text { Dinamómetro: } \\
\text { - Extensor de rodilla } \\
\text { (ER) } \\
\text { - Flexor de rodilla (FR) }\end{array}$ & $\begin{array}{l}\text { TTC: mostró un } \\
\text { aumento estadística- } \\
\text { mente significativo } \\
\text { en: ER excéntrico } \\
\text { (p=0.01). } \\
\text { GC diferencia: } 10.5 \% \\
\text { TC diferencia: } 21.3 \%\end{array}$ \\
\hline $\begin{array}{l}\text { Liv et al. } \\
(2012)^{25}\end{array}$ & $\begin{array}{l}\text { Evaluar el efecto } \\
\text { de un programa de } \\
\text { Tai Chi en compar- } \\
\text { ación con ejercicios } \\
\text { cinestésicos sobre la } \\
\text { fuerza muscular de } \\
\text { tobillos en adultos } \\
\text { mayores. }\end{array}$ & $\begin{array}{l}n=42 \\
60 \text { a } 85 \text { años. } \\
H: 18 \\
M: 24\end{array}$ & $\begin{array}{l}\text { Tai Chi (TC) } \\
\text { (forma 24, } \\
\text { estilo Yang). }\end{array}$ & $\begin{array}{l}45 \text { minutos, } 2 \\
\text { veces por se- } \\
\text { mana durante } 16 \\
\text { semanas. }\end{array}$ & $\begin{array}{l}\text { Ensayo clínico Ensayo } \\
\text { clínico aleatorizado. } \\
\text { Grupos: } \\
\text { TC (n= 15) } \\
\text { Ejercicios cinestésicos (PE) } \\
\text { (2 veces por semana, } \\
16 \text { semanas, } 45 \text { minutos; } \\
\text { ejercicios dinámicos y } \\
\text { estáticos) } \\
\text { (n=10) } \\
\text { Grupo ejercicios no es- } \\
\text { tructurados (GC) } \\
\text { (n=17) }\end{array}$ & $\begin{array}{l}\text { Dinamómetro isociné- } \\
\text { tico: } \\
\text { - Dorsiflexión de tobillo } \\
\text { derecho e izquierdo } \\
\text { (DFTD, DFTI) } \\
\text { - Flexión plantar de } \\
\text { tobillo derecho e izqui- } \\
\text { erdo (FPTD y FPTI) }\end{array}$ & $\begin{array}{l}\text { Sin diferencias es- } \\
\text { tadísticamente signifi- } \\
\text { cativas entre grupos } \\
(p=0.11-0.15)\end{array}$ \\
\hline $\begin{array}{l}\text { Barbat- } \\
\text { Artigas } \\
\text { et al. } \\
(2011)^{42}\end{array}$ & $\begin{array}{l}\text { Determinar el } \\
\text { efecto de un pro- } \\
\text { grama de Tai Chi } \\
\text { en mujeres post- } \\
\text { menopáusicas con } \\
\text { dinapenia tipo } 1 \text { y } \\
\text { sin dinapenia. }\end{array}$ & $\begin{array}{l}n=48 \\
50 \text { a } 75 \text { años. } \\
\text { Grupos: } \\
\text { Con dinap- } \\
\text { enia (CD): } 15 \\
\text { Sin dinap- } \\
\text { enia (SD): } 33 \\
\text { M:48 }\end{array}$ & $\begin{array}{l}\text { Tai Chi (TC) } \\
\text { (forma 24, } \\
\text { estilo Yang) }\end{array}$ & $\begin{array}{l}\text { No mencio- } \\
\text { nan duración } \\
\text { de cada sesión, } \\
\text { mínimo asistían } \\
\text { a } 29 \text { sesiones } \\
\text { durante } \\
12 \text { semanas. }\end{array}$ & $\begin{array}{l}\text { TCuasi-experimental } \\
\text { piloto } \\
\text { TC con dinapenia }(n=15) \\
\text { TC sin dinapenia }(n=33)\end{array}$ & $\begin{array}{l}\text { Bioimpedanciometría } \\
\text { - Masa muscu- } \\
\text { loesquelética (MME) } \\
\text { Dinamómetro: } \\
\text { - Fuerza de agarre (FA } \\
\text { kg/SM kg) }\end{array}$ & $\begin{array}{l}\text { CD: Disminuyó } \\
\text { MME( } p=0.02) \text { y au- } \\
\text { mentó FA }(p=0.04) \\
\text { después de TC. } \\
\text { SD: Sin efecto sig- } \\
\text { nificativo ( } p=0.38 \text { y } \\
p=0.70) \text {. }\end{array}$ \\
\hline
\end{tabular}


Cuadro 1. Características y evidencias de los estudios sobre el efecto del Tai Chi sobre fuerza y masa muscular incluidos en la revisión sistemática

\begin{tabular}{|c|c|c|c|c|c|c|c|}
\hline $\begin{array}{l}\text { Autor/ } \\
\text { año }\end{array}$ & Objetivo & Población & $\begin{array}{l}\text { Tipo de } \\
\text { Tai Chi }\end{array}$ & $\begin{array}{c}\text { Duración, } \\
\text { frecuencia y } \\
\text { tiempo de Tai Chi }\end{array}$ & $\begin{array}{c}\text { Diseño de } \\
\text { investigación }\end{array}$ & Medición & Resultados \\
\hline $\begin{array}{l}\text { Li et al. } \\
(2010)^{31}\end{array}$ & $\begin{array}{l}\text { Determinar el efecto } \\
\text { de la práctica de } \\
\text { Tai Chi modificada } \\
\text { en personas con } \\
\text { neuropatía periférica } \\
\text { sobre la fuerza y fun- } \\
\text { cionalidad física. }\end{array}$ & $\begin{array}{l}n=25 \\
60 \text { a } 80 \text { años. } \\
H: 8 \\
M: 17\end{array}$ & $\begin{array}{l}\text { Tai Chi (TC) } \\
\text { (modificación } \\
\text { de la forma } 8, \\
\text { estilo Yong). }\end{array}$ & $\begin{array}{l}60 \text { minutos, } 3 \\
\text { veces por se- } \\
\text { mana durante } 24 \\
\text { semanas. }\end{array}$ & $\begin{array}{l}\text { Pre-experimental } \\
\text { TC }(n=25)\end{array}$ & $\begin{array}{l}\text { Dinamómetro: } \\
\text { - Extensor de rodilla } \\
\text { (ER) } \\
\text { - Flexor de rodilla (FR) }\end{array}$ & $\begin{array}{l}\text { ER: mostró un aumen- } \\
\text { to estadísticamente } \\
\text { significativo después } \\
\text { de } 6 \text { semanas de TC } \\
(p<0.0001) \text {. }\end{array}$ \\
\hline $\begin{array}{l}\text { Song } \\
\text { etal. } \\
(2010)^{37}\end{array}$ & $\begin{array}{l}\text { Evaluar el efecto del } \\
\text { Tai Chi por } 6 \text { meses } \\
\text { sobre la fuerza mus- } \\
\text { cular de rodilla en } \\
\text { mujeres mayores con } \\
\text { osteoartritis. }\end{array}$ & $\begin{array}{l}n=65 \\
55 \text { a } 70 \text { años. } \\
M: 65\end{array}$ & $\begin{array}{l}\text { Tai Chi (TC) } \\
\text { (forma 31, } \\
\text { estilo Sun) }\end{array}$ & $\begin{array}{l}60 \text { minutos, } 2 \\
\text { veces a la se- } \\
\text { mana durante } 24 \\
\text { semanas. } \\
\text { Los alentaron a } \\
\text { practicarlo en } \\
\text { casa diario por } 20 \\
\text { minutos y regis- } \\
\text { trarlo. }\end{array}$ & $\begin{array}{l}\text { Ensayo clínico aleatorizado. } \\
\text { Grupos: } \\
\text { TC (n=30) } \\
\text { Grupo control (GC) } \\
\text { (n=35) }\end{array}$ & $\begin{array}{l}\text { Dinamómetro isoci- } \\
\text { nético: } \\
\text { - Extensor de rodilla } \\
\text { (ER) } \\
\text { - Flexor de rodilla (FR) }\end{array}$ & $\begin{array}{l}\text { TC: mostró un au- } \\
\text { mento significativa- } \\
\text { mente mayor en ER } \\
\text { (F=6.67, p=0.01) al } \\
\text { compararlos con el } \\
\text { CG. }\end{array}$ \\
\hline $\begin{array}{l}\text { Li et al. } \\
(2009)^{39}\end{array}$ & $\begin{array}{l}\text { Determinar el efecto } \\
\text { de una intervención } \\
\text { de } 16 \text { semanas de Tai } \\
\text { Chi sobre la fuerza } \\
\text { de extremidades } \\
\text { inferiores en adultos } \\
\text { mayores. }\end{array}$ & $\begin{array}{l}n=50 \\
60 \text { a } 70 \text { años. } \\
H: 25 \\
\text { M: } 25\end{array}$ & $\begin{array}{l}\text { Tai Chi (TC) } \\
\text { (forma 24, no } \\
\text { mencionan el } \\
\text { estilo). }\end{array}$ & $\begin{array}{l}60 \text { minutos, } 7 \\
\text { veces por se- } \\
\text { mana durante } 6 \\
\text { semanas. }\end{array}$ & $\begin{array}{l}\text { Ensayo clínico aleatorizado } \\
\text { Grupos: } \\
\text { TC ( } n=32 \text { ) } \\
\text { Grupo control (GC) (se pidió } \\
\text { que no cambiaran su nivel } \\
\text { de actividad física durante el } \\
\text { estudio) ( } n=18 \text { ) }\end{array}$ & $\begin{array}{l}\text { Dinamómetro isoci- } \\
\text { nético: } \\
\text { - Extensor de rodilla } \\
\text { (ER) } \\
\text { - Flexor de rodilla (FR) } \\
\text { - Dorsiflexión de to- } \\
\text { billo (DT) } \\
\text { - Flexión plantar de } \\
\text { tobillo (FPT) }\end{array}$ & $\begin{array}{l}\text { TC: mostró un au- } \\
\text { mento estadística- } \\
\text { mente significativo FR } \\
\text { al comparar con GC } \\
\text { ( } \mathrm{P}=0.046 \text { ). } \\
\text { TC: diferencia } 15.12 \\
\text { GC: diferencia } 7.09\end{array}$ \\
\hline $\begin{array}{l}\text { Xu et al. } \\
(2008)^{38}\end{array}$ & $\begin{array}{l}\text { Valorar el efecto } \\
\text { del Tai Chi sobre la } \\
\text { función muscular en } \\
\text { adultos mayores. }\end{array}$ & $\begin{array}{l}n=61 \\
60 \text { a } 69 \text { años. } \\
M: 25 \\
H: 36\end{array}$ & $\begin{array}{l}\text { Practicantes } \\
\text { Tai Chi (TC) } \\
\text { (ya practi- } \\
\text { caban el es- } \\
\text { tilo Yang, no } \\
\text { mencionan la } \\
\text { forma) }\end{array}$ & $\begin{array}{l}1 \text { hora diaria. } \\
\text { Experiencia de } 4 \\
\text { años. }\end{array}$ & $\begin{array}{l}\text { Cuasi-experimental } \\
\text { Grupos: } \\
\text { TC (n=21) } \\
\text { Grupo corredores (JG) (expe- } \\
\text { riencia de } 4 \text { años o más cor- } \\
\text { riendo por lo menos } 1 \text { hora) } \\
\text { (n= 18) } \\
\text { Grupo control (CG) (sin activi- } \\
\text { dad física por lo menos } 5 \text { años } \\
\text { previos) } \\
\text { (n=22) }\end{array}$ & $\begin{array}{l}\text { Dinamómetro isoci- } \\
\text { nético: } \\
\text { - Extensor de rodilla } \\
\text { (ER) } \\
\text { - Flexor de rodilla (FR) } \\
\text { - Dorsiflexión de to- } \\
\text { billo (DFT) } \\
\text { - Flexión plantar de } \\
\text { tobillo (FPT) }\end{array}$ & $\begin{array}{l}\text { TC: ER mayor resisten- } \\
\text { cia al comparar con } \\
G C(p=0.027) \text {. }\end{array}$ \\
\hline
\end{tabular}


Cuadro 1. Características y evidencias de los estudios sobre el efecto del Tai Chi sobre fuerza y masa muscular incluidos en la revisión sistemática

\begin{tabular}{|c|c|c|c|c|c|c|c|}
\hline $\begin{array}{l}\text { Autor/ } \\
\text { año }\end{array}$ & Objetivo & Población & $\begin{array}{l}\text { Tipo de } \\
\text { Tai Chi }\end{array}$ & $\begin{array}{c}\text { Duración, } \\
\text { frecuencia y } \\
\text { tiempo de Tai Chi }\end{array}$ & $\begin{array}{c}\text { Diseño de } \\
\text { investigación }\end{array}$ & Medición & Resultados \\
\hline $\begin{array}{l}\text { Woo } \\
\text { etal. } \\
(2007)^{15}\end{array}$ & $\begin{array}{l}\text { Medir el efecto de } \\
\text { la práctica del Tai } \\
\text { Chi en compar- } \\
\text { ación con el ejer- } \\
\text { cicio de resistencia } \\
\text { sobre la fuerza } \\
\text { muscular en adultos } \\
\text { mayores de una } \\
\text { comunidad. }\end{array}$ & $\begin{array}{l}n=180 \\
65 \text { a } 74 \text { años. } \\
\text { H: } 90 \\
\text { M: } 90\end{array}$ & $\begin{array}{l}\text { Tai Chi (TC) } \\
\text { (forma 24, } \\
\text { estilo Yang). }\end{array}$ & $\begin{array}{l}\text { No se menciona } \\
\text { el tiempo de eje- } \\
\text { cución por sesión, } \\
3 \text { veces por } \\
\text { semana, durante } \\
52 \text { semanas. }\end{array}$ & $\begin{array}{l}\text { Ensayo clínico aleatori- } \\
\text { zado. } \\
\text { Grupos: } \\
\text { TC (n=60) } \\
\text { Grupo Ejercicio de resist- } \\
\text { encia (RTE) } \\
\text { (3 veces por semana, } \\
\text { ejercicios con bandas de } \\
\text { resistencia) } \\
\text { (n=60) } \\
\text { Grupo control (GC) } \\
\text { (n=60) }\end{array}$ & $\begin{array}{l}\text { Dinamómetro: } \\
\text { - Fuerza de agarre } \\
\text { (FA) } \\
\text { Dinamómetro isociné- } \\
\text { tico: } \\
\text { - Cuádriceps femoral } \\
\text { (CF) }\end{array}$ & $\begin{array}{l}\text { Sin diferencia signifi- } \\
\text { cativa entre grupos } \\
(p=0.45) \text {. }\end{array}$ \\
\hline $\begin{array}{l}\text { Frye } \\
\text { et al. } \\
(2007)^{32}\end{array}$ & $\begin{array}{l}\text { Evaluar el efecto } \\
\text { del Tai Chi en } \\
\text { comparación con } \\
\text { el ejercicio de bajo } \\
\text { impacto sobre la } \\
\text { función física en } \\
\text { adultos mayores. }\end{array}$ & $\begin{array}{l}n=84 \\
52 \text { a } 82 \text { años } \\
H: 30 \\
\text { M: } 54\end{array}$ & $\begin{array}{l}\text { Tai Chi (TC) } \\
\text { (forma 10, } \\
\text { estilo Yang). }\end{array}$ & $\begin{array}{l}60 \text { minutos, } 3 \\
\text { veces por se- } \\
\text { mana durante } 12 \\
\text { semanas. }\end{array}$ & $\begin{array}{l}\text { Ensayo clínico aleatorizado } \\
\text { Grupos: } \\
\text { TC (n=35) } \\
\text { Grupo ejercicio de bajo } \\
\text { impacto (LIE) [3 veces } \\
\text { por semana, } 12 \text { semanas, } \\
60 \text { minutos, ejercicios de } \\
\text { fuerza, flexibilidad, resisten- } \\
\text { cia y balance con bandas } \\
\text { de resistencia] } \\
\text { (n=28) } \\
\text { Grupo control (GC) } \\
\text { (n=21) }\end{array}$ & $\begin{array}{l}\text { Dinamómetro: } \\
\text { - Fuerza de agarre } \\
\text { (FA) }\end{array}$ & $\begin{array}{l}\text { TC y LIE: Aumento FA } \\
\text { en ambos ( } \mathrm{p}<0.05) \text {. } \\
\text { Sin diferencia signifi- } \\
\text { cativa entre grupos. }\end{array}$ \\
\hline $\begin{array}{l}\text { Choi } \\
\text { et al. } \\
(2005)^{33}\end{array}$ & $\begin{array}{l}\text { Determinar el efecto } \\
\text { de la práctica del } \\
\text { Tai Chi sobre la } \\
\text { aptitud física (fuerza } \\
\text { muscular de rodilla } \\
\text { y tobillo) en adultos } \\
\text { mayores institucion- } \\
\text { alizados. }\end{array}$ & $\begin{array}{l}n=59 \\
60 \text { a } 83 \text { años } \\
H: 15 \\
M: 44\end{array}$ & $\begin{array}{l}\text { Tai Chi (TC) } \\
\text { (forma 12, } \\
\text { estilo Sun) }\end{array}$ & $\begin{array}{l}35 \text { minutos, } 3 \\
\text { veces por se- } \\
\text { mana durante } 12 \\
\text { semanas. }\end{array}$ & $\begin{array}{l}\text { Cuasi-experimental } \\
\text { Grupos: } \\
\text { TC (n=29) } \\
\text { Grupo control (GC) } \\
(n=30)\end{array}$ & $\begin{array}{l}\text { Dinamómetro: } \\
\text { - Extensor de rodilla } \\
\text { ( R) } \\
\text { - Flexor de rodilla (FR) } \\
\text { - Dorsiflexión de tobillo } \\
\text { (DFT) } \\
\text { - Flexión plantar de } \\
\text { tobillo (FPT) }\end{array}$ & $\begin{array}{l}\text { TC: aumento signifi- } \\
\text { cativo en FR, DFTy FPT } \\
\text { ( } p<0.001 \text { ) y ER ( } p \\
<0.01 \text { ) al compararlo } \\
\text { con GC. }\end{array}$ \\
\hline
\end{tabular}


Cuadro 1. Características y evidencias de los estudios sobre el efecto del Tai Chi sobre fuerza y masa muscular incluidos en la revisión sistemática

\begin{tabular}{|c|c|c|c|c|c|c|c|}
\hline $\begin{array}{l}\text { Autor/ } \\
\text { año }\end{array}$ & Objetivo & Población & $\begin{array}{l}\text { Tipo de } \\
\text { Tai Chi }\end{array}$ & $\begin{array}{c}\text { Duración, } \\
\text { frecuencia y } \\
\text { tiempo de Tai Chi }\end{array}$ & $\begin{array}{c}\text { Diseño de } \\
\text { investigación }\end{array}$ & Medición & Resultados \\
\hline $\begin{array}{l}\text { Audette } \\
\text { et al. } \\
(2006)^{34}\end{array}$ & $\begin{array}{l}\text { Evaluar el efecto del } \\
\text { Tai Chi modificado } \\
\text { en comparación la } \\
\text { caminata rápida } \\
\text { sobre la fuerza } \\
\text { muscular en mujeres } \\
\text { mayores. }\end{array}$ & $\begin{array}{l}n=27 \\
65 \text { a } 76 \text { años } \\
M: 27\end{array}$ & $\begin{array}{l}\text { Tai Chi (TC) } \\
\text { (forma } 10 \\
\text { modificada, } \\
\text { estilo Yang). }\end{array}$ & $\begin{array}{l}60 \text { minutos, } 3 \\
\text { veces por se- } \\
\text { mana durante } 12 \\
\text { semanas. }\end{array}$ & $\begin{array}{l}\text { Ensayo clínico aleatorizado } \\
\text { Grupos: } \\
\text { TC (n=11) } \\
\text { Grupo caminata rápida } \\
\text { (BWG) [3 veces por semana, } \\
12 \text { semanas, } 1 \text { hora] } \\
\text { (n=8) } \\
\text { Grupo sedentario (GC) } \\
\text { (n=8) }\end{array}$ & $\begin{array}{l}\text { Dinamómetro: } \\
\text { - Fuerza de agarre } \\
\text { (FAD y FA I) } \\
\text { - Extensor de rodilla } \\
\text { derecho e izquierdo } \\
\text { (ERD, ERI) }\end{array}$ & $\begin{array}{l}\text { TC y BWG: se observó } \\
\text { un aumento significa- } \\
\text { tivo ERI ( } p=0.04) \text {. }\end{array}$ \\
\hline $\begin{array}{l}\text { Wu } \\
\text { etal. } \\
(2002)^{25}\end{array}$ & $\begin{array}{l}\text { Medir la fuerza isoci- } \\
\text { nética de músculos } \\
\text { de la pierna entre } \\
\text { practicantes a largo } \\
\text { plazo de Tai Chi. }\end{array}$ & $\begin{array}{l}n=39 \\
60 \text { a } 65 \text { años } \\
H: 19 \\
M: 20\end{array}$ & $\begin{array}{l}\text { Practicantes } \\
\text { Tai Chi (TC) }\end{array}$ & $\begin{array}{l}1 \text { hora, } 3 \text { veces } \\
\text { por semana, ya } \\
\text { practicaban Tai } \\
\text { Chi por mínimo } 3 \\
\text { años. }\end{array}$ & $\begin{array}{l}\text { Transversal analítico } \\
\text { Grupos: } \\
\text { TC(n=20) } \\
\text { Grupo control (GC) (nunca } \\
\text { habían practicado Tai Chi } \\
\text { antes, pero eran físicamente } \\
\text { activos) } \\
\text { (n=19) }\end{array}$ & $\begin{array}{l}\text { Dinamómetro isoci- } \\
\text { nético: } \\
\text { - Extensor de rodilla } \\
\text { (ER) } \\
\text { - Flexor de rodilla (FR) }\end{array}$ & $\begin{array}{l}\text { TC: diferencia } \\
\text { significativa ER en } \\
\text { hombres y mujeres } \\
\text { al comparar con GC } \\
(p<0.05) \text {. }\end{array}$ \\
\hline $\begin{array}{l}\text { Lan } \\
\text { etal. } \\
(2000)^{40}\end{array}$ & $\begin{array}{l}\text { Evaluar el efecto de } \\
\text { un programa de Tai } \\
\text { Chi sobre la fuerza } \\
\text { muscular del extensor } \\
\text { de rodilla en adultos } \\
\text { mayores. }\end{array}$ & $\begin{array}{l}n=32 \\
55 \text { a } 70 \text { años. } \\
H: 15 \\
M: 17\end{array}$ & $\begin{array}{l}\text { Tai Chi (TC) } \\
\text { (no menciona } \\
\text { la forma, es- } \\
\text { tilo Yang). }\end{array}$ & $\begin{array}{l}60 \text { minutos, } 7 \\
\text { veces por se- } \\
\text { mana durante } 24 \\
\text { semanas. }\end{array}$ & Pre-experimental & $\begin{array}{l}\text { Dinamómetro isomé- } \\
\text { trico: } \\
\text { - Extensor de rodilla } \\
\text { derecho e izquierdo } \\
\text { (ERD, ERI) }\end{array}$ & $\begin{array}{l}\text { Diferencias significa- } \\
\text { tivas pre y post test, } \\
\text { en mujeres y hom- } \\
\text { bres, en ERD y ERI de } \\
\text { pierna dominante } \\
\text { (p<0.05). }\end{array}$ \\
\hline $\begin{array}{l}\text { Wolf } \\
\text { et al. } \\
(1996)^{41}\end{array}$ & $\begin{array}{l}\text { Determinar el efecto } \\
\text { del Tai Chi en com- } \\
\text { paración con el } \\
\text { entrenamiento de } \\
\text { balance computari- } \\
\text { zado sobre la fuerza } \\
\text { de agarre. }\end{array}$ & $\begin{array}{l}n=280 \\
70 \text { a } 80 \text { años. } \\
H: 58 \\
\text { M: } 222\end{array}$ & $\begin{array}{l}\text { Tai Chi (TC) } \\
\text { (no mencio- } \\
\text { nan forma ni } \\
\text { estilo, ya que } \\
\text { fue práctica } \\
\text { en casa sin } \\
\text { monitoreo). }\end{array}$ & $\begin{array}{l}30 \text { minutos, } 7 \\
\text { días a la semana } \\
\text { durante } 15 \text { sema- } \\
\text { nas. }\end{array}$ & $\begin{array}{l}\text { Ensayo clínico aleatorizado } \\
\text { Grupos: } \\
\text { TC (n=152) } \\
\text { Grupo entrenamiento de } \\
\text { balance computarizado (BT) } \\
\text { (n=64) } \\
\text { Grupo de educación (ED) (sin } \\
\text { cambios en su actividad física } \\
\text { y tuvieron } 1 \text { vez a la semana } \\
\text { por } 1 \text { hora una sesión informa- } \\
\text { tiva) (n=64) }\end{array}$ & $\begin{array}{l}\text { Dinamómetro: } \\
\text { - Fuerza de agarre } \\
\text { (FA) }\end{array}$ & $\begin{array}{l}\text { BT y ED: disminución } \\
\text { de FA al compararlos } \\
\text { con TC ( } p=0.0249), \\
\text { por lo que hay } \\
\text { menos pérdida de } \\
\text { fuerza en TC. }\end{array}$ \\
\hline
\end{tabular}

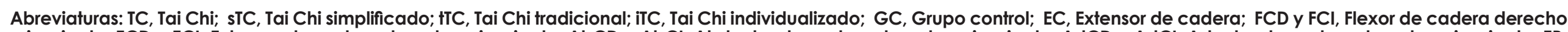

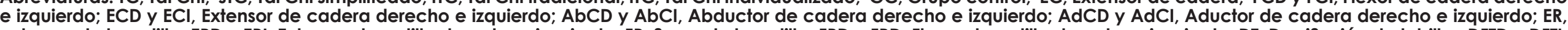

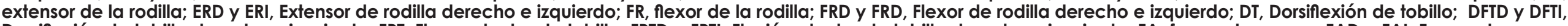

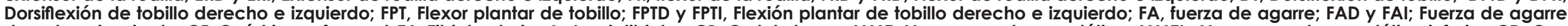

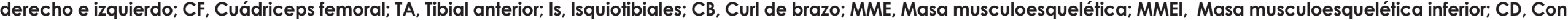
dinapenia; SD, Sin dinapenia 
A partir de la evidencia obtenida, 6 de los 25 estudios llevaron a cabo solamente la medición de la fuerza muscular en miembros superiores, $22,23,32,41-43$ de los cuales en dos se observaron cambios positivos estadísticamente significativos $(p<0.05)$ respecto a flexibilidad, fuerza y movilidad al ser comparadas las mediciones antes y depués de la intervención, ya que ambos son estudios pre-experimentales ( $\sin$ grupo control). ${ }^{22,23}$ En los resultados obtenidos por Wolf et al. (1996) ${ }^{41}$ y Hwang et al. (2016) ${ }^{43}$ no se observó ningún cambio significativo al comparar TC con diferentes tipos de ejercicios. En 16 estudios se realizó la medición de la fuerza de miembros inferiores, ${ }^{21,24-31,33,36-40,45} 14$ mostraron cambios positivos estadísticamente significativos $(p<0.05)$ en la fuerza muscular y de los tendones de tobillos, rodillas y cadera, además de otras variables como la mejora del equilibrio y seguridad en marcha. Chen et al. $(2020)^{27}$ y Penn et al. (2019) ${ }^{28}$ reportaron una mayor eficacia cuando las personas realizan una rutina de TC personalizada a diferencia de sesiones grupales. Por otro lado en cuatro estudios, no se encontró ningún tipo de beneficio respecto a fuerza, sin embargo, Liu et al. (2012) ${ }^{25}$ resaltan una mejor percepción de salud y satisfacción por parte de su grupo que practicó TC. Asimismo, en 11 de los 16 estudios compararon al grupo de TC con un grupo control, eran estudios pre-experimentales o de cohorte. $21,24,26,29-31,33,37,39,40,45$ La mayoría de los que se compararon con otro tipo de actividad física como caminar, correr, bailar o ejercicio no estructurado, no mostraron ninguna diferencia estadísticamente significativa en comparación con el entrenamiento de TC ( $p>0.05)$, en la fuerza del tren inferior. ${ }^{25,27,28,36,38,44}$

Es importante resaltar que solamente en dos estudios se evaluó la fuerza de miembros inferiores y superiores, ${ }^{15,34}$ aunque en ambos estudios no se encontraron diferencias estadísticamente significativas entre el entrenamiento de TC en comparación con otros ejercicios.

\section{MASA MUSCULAR}

En tres estudios se midió la cantidad de masa muscular por medio de BIA. ${ }^{23,42,44}$ En este sentido, solamente en dos se observó aumento de la masa musculoesquelética, ${ }^{23,44}$ aunque uno es un estudio de cohorte en el que se observó un incremento estadísticamente significativo $(p<0.05)$ en la masa muscular con la práctica de TC con una duración de 60 minutos, dos veces por semana durante 16 semanas. ${ }^{23}$
Asimismo, en el otro estudio (cuasi-experimental) se encontró un incremento estadísticamente significativo $(p<0.05)$ en la masa muscular en el grupo que practicó TC (60 minutos, cinco veces por semana durante seis semanas) en comparación con el grupo de sedentarios ${ }^{44}$ Paradójicamente en el otro estudio (cuasi-experimental piloto) se observó una disminución en la masa muscular $(16.31 \pm 2.83$ vs. 15.48 $\pm 2.56, p=0.02$ ) después de la práctica de TC estilo Yang durante 12 semanas en un grupo de mujeres que presentaban dinapenia en comparación otro sin dinapenia. ${ }^{42}$

\section{TIPOS DE EJERCICIO FÍSICO}

En la mayoría de estudios se especifica la forma y el estilo de TC que se practicó. Al respecto, el estilo predominante fue Yang con la forma 24. En seis de estos estudios se observaron resultados positivos con respecto a la práctica de TC. ${ }^{15,25,28,35,42,44}$ No obstante, en cuatro estudios no reportaron el estilo ni la forma de TC..$^{21,24,26,41}$

\section{Discusión}

EI TC es un ejercicio moderado y seguro, recomendado para personas mayores de 60 años. De los 25 estudios analizados, en 20 se encontró un efecto positivo sobre la fuerza muscular de miembros superiores e inferiores cuando se practicó el TC por más de 8 semanas, durante una hora, aunque no se precisa el número de días. Asimismo, se observó que el TC tiene mayor impacto en miembros inferiores, aunque algunos estudios encontraron un aumento en la fuerza de agarre. Sin embargo, al compararlo con algún tipo de actividad física diferente, el cambio no fue significativo. Con relación a la masa muscular solo en tres estudios se midió esta variable, de los cuales en dos se encontró un efecto positivo sobre la masa muscular, aunque el diseño de uno de ellos fue de cohorte. Por otro lado, en el tercer estudio se encontró una disminución estadísticamente significativa después del entrenamiento del TC en mujeres con dinapenia.

FUERZA

EI TC es un ejercicio que trabaja en su mayoría la parte inferior del cuerpo debido a la postura en semiflexión de las rodillas que requiere para realizar los diversos movimientos. ${ }^{27}$ En este sentido, los estudios analizados en este trabajo muestran que también 
hay un beneficio generalizado en la fuerza de todo el cuerpo, aunque, la mayoría demostraron que hay cambios mayores en la fuerza de miembros inferiores.

El análisis incluyó ensayos clínicos, estudios experimentales y de cohorte, cuyos resultados sugieren que el TC es una buena opción para mantener la fuerza existente en personas en proceso de envejecimiento. ${ }^{22,28,29,35,42-44}$ Algunos estudios incluyen personas con enfermedades como dinapenia o artritis, en quienes se observó mejores resultados en comparación con personas sanas. Asimismo, los resultados de dichos estudios sugieren que la práctica de TC, realizado bajo supervisión, el estilo adecuado, con un tiempo mínimo de 12 meses, puede ayudar más a personas con alguna condición patológica para mejorar la fuerza muscular. ${ }^{21,23,27,36}$

Otro aspecto que se debe tomar en cuenta es que en dos estudios se reportó que el TC personalizado muestra mejores resultados que el de tipo grupal (tradicional), ${ }^{24,35}$ esto puede deberse a que se toman en cuenta las características, habilidades y posibilidades de los participantes para llevar a cabo el ejercicio. No obstante, a nivel comunitario, esta opción no es viable del todo, debido al número de participantes que practican el TC en la comunidad, aunque, es importante tomarlo en cuenta para evaluar la eficacia de ciertas posturas y movimientos en cuanto al mantenimiento de la fuerza muscular en casos específicos.

Al comparar TC con otro tipo ejercicio físico, no se observaron diferencias estadísticamente significativas respecto a la fuerza muscular, ${ }^{15,25,30,31,38,39,42,43,46}$ esto puede ser debido al esfuerzo que demanda realizar otras actividades como caminata rápida comparado con un ejercicio con movimientos lentos y controlados. Estos resultados sugieren que el TC puede ser una opción para mantener la fuerza muscular durante un tiempo limitado, aunque es recomendable un progreso a otro tipo de actividad física que requiera mayor demanda de esfuerzo, para poder aumentar la fuerza muscular en personas en proceso de envejecimiento. Es importante destacar que solamente tres estudios $^{22,36,42}$ evaluaron la intensidad del ejercicio, este componente es importante para poder realizar una comparación completa entre ejercicios y saber a qué intensidades y frecuencias se debe llevar a cabo, para que se observe un beneficio en la cantidad y calidad muscular.

\section{MASA MUSCULAR}

Es escasa la evidencia científica respecto al efecto del entrenamiento de TC sobre la masa muscular. En este sentido, solo se encontraron tres estudios (doscuasi-experimentales y uno de cohorte) en los que utilizó el mismo estilo y movimientos de TC y midieron el efecto sobre la masa muscular, sin embargo, ninguno es un ensayo clínico aleatorizado, tampoco se estimó el tamaño de la muestra. Asimismo, los diseños de investigación son muy heterogéneos, con resultados inconsistentes, ya que incluso en uno de ellos se encontró una disminución de la masa muscular en un grupo de mujeres con dinapenia. ${ }^{42}$ Otro de los estudios es de cohorte ${ }^{23}$ por lo que no puede ser comparado con los otros dos estudios (cuasi-experimentales). ${ }^{42,44}$ Por tal, motivo es necesario llevar a cabo más investigación, preferentemente ensayos clínicos aleatorizados, en los que la intervención sea similar respecto a tipo, frecuencia y tiempo, así como la medición de la fuerza y masa muscular con equipos confiables.

\section{TIPOS DE EJERCICIOS}

Es importante recalcar que algunos autores no mencionaron la forma ni estilo que llevaron a cabo como intervención, en la mayoría de los casos la frecuencia e intensidad de las mismas rutinas tampoco fue explícita: En este sentido, Wong et al. ${ }^{47}$ señalan que el efecto del TC en general es satisfactorio, aunque es necesario estandarizar rutinas y planes de seguimiento para encontrar resultados óptimos en las investigaciones.

Los estilos de TC que más se especificaron en los estudios fueron "Yang" y "Sun", esto puede ser debido a que las posturas y los movimientos son más lentos y gentiles, lo que hace que la práctica sea segura para los participantes. Especialmente el estilo "Sun" se recomienda para personas mayores con alguna condición clínica como artritis. ${ }^{22}$ Finalmente, en ninguno de los estudios revisados se reportaron riesgos o cambios desfavorables en las poblaciones estudiadas que practicaron TC. 
Entre las limitaciones más importantes del estudio se pueden señalar la heterogeneidad metodológica de estudios seleccionados que cumplieron los criterios de elegibilidad, lo cual no permitió valorar con precisión el efecto del entrenamiento del TC sobre la fuerza y masa muscular durante el proceso de envejecimiento. Por tal motivo, es necesario llevar a cabo más investigación con una metodología rigurosa y entrenamiento de TC homogéneo en cuanto a tipo, tiempo y frecuencia, para poder realizar una RS más precisa con posibilidades de un meta-análisis, con el propósito de valorar el efecto del entrenamiento del TC para la prevención y control de la sarcopenia.

\section{Conclusiones}

EI TC es un ejercicio físico moderado seguro que podría tener un efecto positivo para el mantenimiento de fuerza al igual que el ejercicio de resistencia, sin embargo, las evidencias son limitadas y no concluyentes, por tal motivo, es necesario llevar a cabo más ensayos clínicos o estudios cuasi-experimentales con una metodología rigurosa, considerando diferentes tipos y tiempo de entrenamiento de TC, en diferentes grupos de edad (35-44 años, 45-59 años, 60-74 años, 75-89 años) y estados de salud (diabetes mellitus, obesidad, hipertensión arterial en comparación con sanos).

\section{Agradecimientos}

El estudio se llevó a cabo con el apoyo financiero de la Dirección General de Asuntos del Personal Académico, UNAM, proyecto PAPIIT IN306121. También agradecemos la asesoría metodológica de la Red Académica Asesora de Revisiones Sistemáticas (RAARS) de la FES Zaragoza, UNAM.

\section{Referencias}

1. Martínez-Maldonado ML, Mendoza-Núñez VM. Promoción de la salud de la mujer adulta mayor. Ciudad de México: Instituto Nacional de Geriatría; 2015.

2. González Rodríguez R, Cardentey García J, Hernández Díaz D de la $C$, Rosales Álvarez G, Jeres Castillo CM. Comportamiento de la fragilidad en adultos mayores TT - Fragility in the elderly. Arch Med Camaguey. 2017;21(4):498-509.

3. Anker SD, Morley JE, von Haehling S. Welcome to the ICD-10 code for sarcopenia. J Cachexia Sarcopenia Muscle. 2016;7(5):512-514.

4. Aelbrecht, S, Beckwée, D DA. Exercise interventions for the prevention and treatment of sarcopenia. A systemic umbrella review. J Nutr Heal Aging. 2019;23(6):495-502.

5. Labata-Lezaun N, Llurda-Almuzara L, López-Decelis C, Rodríguez-Sanz J, González-Rueda V, Hidalgo-García C, et al. Effectiveness of protein supplementation combined with resistance training on muscle strength and physical performance in elderly: A systematic review and meta-analysis. Nutrients. 2020;12:1-16.

6. Martone AM, Marzetti E, Salini S, Zazzara MB, Santoro L, Tosato M, et al. Sarcopenia identified according to the EWGSOP2 definition in communityliving people: prevalence and clinical features. J Am Med Dir Assoc. 2020;21(10):1470-1474.

7. Shafiee G, Keshtkar A, Soltani A, Ahadi Z, Larijani $B$, Heshmat R. Prevalence of sarcopenia in the world: a systematic review and meta- analysis of general population studies. J Diabetes Metab Disord. 2017;16:21. doi: 10.1186/s40200-0170302-x. Available from: https://link.springer.com/ article/10.1186\%2Fs40200-017-0302-X

8. Curcio F, Ferro G, Basile C, Liguori I, Parrella P, Pirozzi $F$, et al. Biomarkers in sarcopenia: a multifactorial approach. Exp Gerontol. 2016;85:1-8. doi: 10.1016/j.exger.2016.09.007.

9. Solano-García W, Carazo-Vargas P. Intervenciones con ejercicio contra resistencia en las personas adultas mayores diagnosticadas con sarcopenia. Una revisión sistemática. PENSAR EN MOVIMIENTO: Revista de Ciencias del Ejercicio y la Salud. 2018;16: e3000. doi.org/10.15517/pensarmov.v16i1.3000.

10. Liguori I, Russo G, Aran L, Bulli G, Curcio F, Della-Morte D, et al. Sarcopenia: sssessment of disease burden and strategies to improve outcomes. Clin 


\section{Casos y revisiones \\ de salud}

Interv Aging. 2018;13:913-927. doi: 10.2147/CIA. S149232. eCollection 2018.

11. Navarro ML, Leiva ML, Narváez VPD, Orellana EA. Efectos de un programa de ejercicios para evaluar las capacidades funcionales y el balance de un grupo de adultos mayores independientes sedentarios que viven en la comunidad. Salud Uninorte. 2011;27(2):185-197.

12. Suksom D, Sirsipatt A, Lapo P, Patumraj S. Effects of two modes of exercise on physical fitness and endothelial function in the elderly: Exercise with a flexible stick versus Tai Chi. J Med Assoc Thail. 2011;94(1):123-132.

13. Adler PA, Roberts BL. The use of Tai Chi to improve health in older adults. Orthop Nurs. 2006;25(2):122126.

14. Wu S, Chen J, Wang S, Jiang M, Wang X, Wen Y. Effect of tai chi exercise on balance function of stroke patients: a meta-analysis. Med Sci Monit Basic Res. 2018;24:210-215.

15. Woo J, Hong A, Lau E, Lynn H. A randomised controlled trial of tai chi and resistance exercise on bone health, muscle strength and balance in communityliving elderly people. Age Ageing. 2007;36(3):262268.

16. Wayne PM, Manor B, Novak V, Costa MD, Hausdorff JM, Goldberger AL, et al. A systems biology approach to studying tai chi, physiological complexity and healthy aging: design and rationale of a pragmatic randomized controlled trial. Contemp Clin Trials. 2013;34(1):21-34.

17. Maris SA, Quintanilla D, Taetzsch A, Picard A, Letendre J, Mahler L, Lofgren I, Xu F, Delmonico MJ. The combined effects of tai chi, resistance training, and diet on physical function and body composition in obese older women. J Aging Res. 2014;2014:657851. doi: $10.1155 / 2014 / 657851$.

18. Pirauá ALT, Cavalcante BR, de Oliveira VMA, Beltrão NB, de Amorim Batista G, Pitangui ACR, et al. Effect of 24-week strength training on unstable surfaces on mobility, balance, and concern about falling in older adults. Scand J Med Sci Sport. 2019;29(11):1805-1812.

19. Zech A, Drey M, Freiberger E, Hentschke C, Bauer JM, Sieber CC, et al. Residual effects of muscle strength and muscle power training and detraining on physical function in community-dwelling prefrail older adults: a randomized controlled trial. BMC Geriatr. 2012;12:68. doi: 10.1186/1471-2318-12-68.

20. Moher D, Liberati A, Tetzlaff J, Altman DG. Preferred reporting items for systematic reviews and metaanalyses: the PRISMA statement. Int J Surg 2010; 8(5):336-341.

21. Bubela D, Sacharko L, Chan J, Brady M. Balance and functional outcomes for older communitydwelling adults who practice tai chi and those who do not: a comparative study. J Geriatr Phys Ther. 2019;42(4):209-215.

22. Dogra S, Shah S, Patel M, Tamim H. Effectiveness of a tai chi intervention for improving functional fitness and general health among ethnically diverse older adults with self-reported arthritis living in low-income neighborhoods: a cohort study. J Geriatr Phys Ther. 2015;38(2):71-77.

23. Manson J, Rotondi M, Jamnik V, Ardern C, Tamim $\mathrm{H}$. Effect of tai chi on musculoskeletal health-related fitness and self-reported physical health changes in low income, multiple ethnicity mid to older adults. BMC Geriatr. 2013;13:114. doi: 10.1186/1471-2318$13-114$

24. Lu X, Hui-Chan CWY, Tsang WWN. Tai chi, arterial compliance, and muscle strength in older adults. Eur J Prev Cardiol. 2013;20(4):613-619.

25. Liu J, Wang XQ, Zheng JJ, Pan YJ, Hua YH, Zhao SM, et al. Effects of tai chi versus proprioception exercise program on neuromuscular function of the ankle in elderly people: a randomized controlled trial. Evid Based Complement Alternat Med. 2012;2012:265486. doi: 10.1155/2012/265486.

26. Wu G, Zhao F, Zhou X, Wei L. Improvement of isokinetic knee extensor strength and reduction of postural sway in the elderly from long-term Tai Chi 
exercise. Arch Phys Med Rehabil. 2002;83(10):13641369.

27. Chen PJ, Penn IW, Wei SH, Chuang LR, Sung $\mathrm{WH}$. Augmented reality-assisted training with selected Tai-Chi movements improves balance control and increases lower limb muscle strength in older adults: a prospective randomized trial. J Exerc Sci Fit. 2020;18(3):142-147.

28. Penn IW, Sung $\mathrm{WH}$, Lin $\mathrm{CH}$, Chuang E, Chuang TY, Lin PH. Effects of individualized Tai-Chi on balance and lower-limb strength in older adults. BMC Geriatr. 2019;19(1):235. doi: 10.1186/s12877-0191250-8.

29. Sung WH, Liu CC, Wei SH, Chuang LR, Chuang $\mathrm{E}$, Wang KA, et al. Feasibility and outcome of an individualized Tai Chi program for improving balance and strength in the elderly: a pilot study. NeuroRehabilitation. 2019;20(4):10-20.

30. Lu X, Hui-Chan CWY, Tsang WWN. Effects of tai chi training on arterial compliance and muscle strength in female seniors: a randomized clinical trial. Eur J Prev Cardiol. 2013;20(1):238-245.

31. Li L, Manor B. Long term tai chi exercise improves physical performance among people with peripheral neuropathy. Am J Chin Med. 2010;38(3):449-459.

32. Frye B, Scheinthal S, Kemarskaya T, Pruchno R. Tai chi and low impact exercise: Effects on the physical functioning and psychological well-being of older people. J Appl Gerontol. 2007;26(5):433-453.

33. Choi JH, Moon JS, Song R. Effects of sun-style tai chi exercise on physical fitness and fall prevention in fall-prone older adults. J Adv Nurs. 2005;51(2):150157.

34. Audette JF, Jin YS, Newcomer R, Stein L, Duncan G, Frontera WR. Tai chi versus brisk walking in elderly women. Age Ageing. 2006;35(4):388-393.

35. Zhou M, Peng N, Dai Q, Li HW, Shi RG, Huang W. Effect of tai chi on muscle strength of the lower extremities in the elderly. Chin J Integr Med.
2016;22(11):861-866. doi: 10.1007/s11655-0152104-7.

36. Song $\mathrm{QH}$, Zhang QH, Xu RM, Ma M, Zhao XP, Shen $G Q$, et al. Effect of tai chi exercise on lower limb muscle strength, bone mineral density and balance function of elderly women. Int J Clin Exp Med. 2014;7(6):1569-1576.

37. Song R, Roberts BL, Lee EO, Lam P, Bae SC. A randomized study of the effects of t'ai chi on muscle strength, bone mineral density, and fear of falling in women with osteoarthritis. J Altern Complement Med. 2010;16(3):227-233.

38. Xu D, Hong Y, Li J. Tai chi exercise and muscle strength and endurance in older people. Med Sport Sci. 2008;55:20-29.

39. Li JX, Xu DQ, Hong Y. Changes in muscle strength, endurance, and reaction of the lower extremities with tai chi intervention. J Biomech. 2009;42(8):967-971.

40. Lan C, Lai JS, Chen SY, Wong MK. Tai Chi Chuan to improve muscular strength and endurance in elderly individuals: a pilot study. Arch Phys Med Rehabil. 2000;81(5):604-607.

41. Wolf SL, Barnhart HX, Kutner NG, McNeely E, Coogler C, Xu T. Reducing frailty and falls in older persons: an investigation of tai chi and computerized balance training. J Am Geriatr Soc. 1996;44(5):489497.

42. Barbat-Artigas S, Filion ME, Dupontgand S, Karelis $A D$, Aubertin-Leheudre M. Effects of tai chi training in dynapenic and nondynapenic postmenopausal women. Menopause. 2011;18(9):974-979.

43. Hwang HF, Chen SJ, Lee-Hsieh J, Chien DK, Chen CY, Lin MR. Effects of home-based tai chi and lower extremity training and self-practice on falls and functional outcomes in older fallers from the emergency department-A randomized controlled trial. J Am Geriatr Soc. 2016;64(3):518-525. doi: 10.1111/ jgs.13952. 


\section{Casos y revisiones \\ de salud

44. Chunguk $\mathrm{O}$, Kang $\mathrm{H}$. Effects of tai chi exercise on the body composition, self-efficacy and life satisfaction of older adults in Korean local community. Int J Gerontol. 2019;13(2):134-138.

45. Chang S, Zhou J, Hong $Y$, Sun W, Cong $Y$, Qin $M$, et al. Effects of 24-week tai chi exercise on the knee and ankle proprioception of older women. Res Sports Med. 2016;24(1):84-93. doi: 10.1080/15438627.2015.1126281.

46. Song R, Roberts BL, Lee EO, Lam P, Bae SC. A randomized study of the effects of t'ai chi on muscle strength, bone mineral density, and fear of falling in women with osteoarthritis. J Altern Complement Med. 2010;16(3):227-233.

47. Wong AM, Lan C. Tai chi and balance control. Med Sport Sci. 2008;52:115-123.

48. Son NK, Ryu YU, Jeong HW, Jang YH, Kim HD. Comparison of 2 different exercise approaches: tai chi versus otago, in community-dwelling older women. J Geriatr Phys Ther. 2016;39(2):51-57.

49. Wu G. Muscle action pattern and knee extensor strength of older Tai Chi exercisers. Med Sport Sci. 2008;52:30-39. doi: 10.1159/000134282.

50. Chen YS, Crowley Z, Zhou S, Cartwright C. Effects of 12-week tai chi training on soleus H-reflex and muscle strength in older adults: a pilot study. Eur J Appl Physiol. 2012;112(6):2363-2368. doi: 10.1007/ s00421-011-2182-y.

51. Murphy L, Singh BB. Effects of 5-form, yang style tai chi on older females who have or are at risk for developing osteoporosis. Physiother Theory Pract. 2008;24(5):311-320. doi: $10.1080 / 09593980701884790$.

52. Qi M, Moyle W, Jones C, Weeks B. Tai chi combined with resistance training for adults aged 50 years and older: a systematic review. J Geriatr Phys Ther. 2020;43:32-41.

53. Gallagher B. Tai Chi Chuan and Qigong: Physical and mental practice for functional mobility. Top Geriatr Rehabil. 2003;19(3):172-182.
54. Kuo LT, Hsu RW-W, Hsu W-H, Lin Z-R. The Comparison of Impact of Circuit Exercise Training and TaiChi Exercise on Multiple Fracture-Related Risk Factors in Postmenopausal Osteopenic Women. J Clin Densitom. 2014 Jul;17(3):427-428.

55. Solloway MR, Taylor SL, Shekelle PG, Miake-Lye IM, Beroes JM, Shanman RM, et al. An evidence map of the effect of tai chi on health outcomes. Syst Rev. 2016;5(1):126. doi: 10.1186/s13643-016-0300-y.

56. Callahan LF, Cleveland RJ, Altpeter M, Hackney B. Evaluation of tai chi program effectiveness for people with arthritis in the community: a randomized controlled trial. J Aging Phys Act. 2016;24(1):101110. doi: 10.1123/japa.2014-0211.

57. Gow BJ, Hausdorff JM, Manor B, Lipsitz LA, Macklin EA, Bonato $P$, et al. Can tai chi training impact fractal stride time dynamics, an index of gait health, in older adults? Cross-sectional and randomized trial studies. PLoS One. 2017;12(10):e0186212. doi: 10.1371/journal.pone.0186212.

58. Yıldırım P, Ofluoglu D, Aydogan S, Akyuz G. Tai Chi vs. combined exercise prescription: a comparison of their effects on factors related to falls. J Back Musculoskelet Rehabil. 2016;29(3):493-501. doi: 10.3233/BMR-150645.

59. Lee HY. [Comparison of effects among Tai-Chi exercise, aquatic exercise, and a self-help program for patients with knee osteoarthritis]. Taehan Kanho Hakhoe Chi. 2006;36(3):571-580. Korean. doi: 10.4040/jkan.2006.36.3.571.

60. Li F, Harmer P, Fitzgerald K, Eckstrom E, Akers L, Chou LS, et al. Effectiveness of therapeutic Tai Ji Quan intervention vs a multimodal exercise intervention to prevent falls among older adults at high risk of falling: a randomized clinical trial. JAMA Intern Med. 2018;178(10):1301-1310. doi: 10.1001/jamainternmed.2018.3915.

61. Li F, Harmer P, Eckstrom E, Fitzgerald K, Chou LS, Liu Y. Effectiveness of Tai Ji Quan vs Multimodal and Stretching Exercise Interventions for Reducing Injurious Falls in Older Adults at High Risk of Falling: Follow-up Analysis of a Randomized Clinical Trial. 
JAMA Netw Open. 2019;2(2):e188280. doi: 10.1001/ jamanetworkopen.2018.8280.

62. Rosado-Pérez J, Santiago-Osorio E, Ortiz R, Mendoza-Núñez VM. Tai chi diminishes oxidative stress in Mexican older adults. J Nutr Heal Aging. 2012;16(7):642-646.

63. Sun W, Ma X, Wang L, Zhang C, Song Q, Gu H, et al. Effects of Tai Chi Chuan and brisk walking exercise on balance ability in elderly women: a randomized controlled trial. Motor Control. 2019;23(1):100-114. doi: 10.1123/mc.2017-0055.

64. Kienle GS, Werthmann PG, Grotejohann B, Kaier $\mathrm{K}$, Steinbrenner I, Voigt-Radloff S, et al. A multi-centre, parallel-group, randomised controlled trial to as- sess the efficacy and safety of eurythmy therapy and tai chi in comparison with standard care in chronically ill elderly patients with increased risk of falling (ENTAiER): a trial protocol. BMC Geriatr. 2020;20(1):108. doi: 10.1186/s12877-020-1503-6.

65. Mihay LM, Boggs KM, Breck AJ, Dokken EL, NaThalang GC. The effect of tai chi inspired exercise compared to strength training: a pilot study of elderly retired community dwellers. Phys Occup Ther Geriatr. 2006; 24:3, 13-26. doi: 10.1080/J148v24n03_02

66. Pereira MM, de Oliveira RJ, Silva MAF, Souza LHR, Vianna LG. Effects of Tai Chi Chuan on knee extensor muscle strength and balance in elderly women. Rev Bras Fisioter. 2008;12(2):121-126. 